\title{
THE
}

$11-22-2004$

\section{Broken-Symmetry Unrestricted Hybrid Density Functional Calculations on Nickel Dimer and Nickel Hydride}

Cristian V. Diaconu

Art E. Cho

J. D. Doll

David L. Freeman

University of Rhode Island, dfreeman@uri.edu

Follow this and additional works at: https://digitalcommons.uri.edu/chm_facpubs

Terms of Use

All rights reserved under copyright.

\section{Citation/Publisher Attribution}

Diaconu, C. V., Choi, A. E., Doll, J. D., \& Freeman, D. L. (2004). Broken-Symmetry Unrestricted Hybrid Density Functional Calculations on Nickel Dimer and Nickel Hydride. Journal of Chemical Physics, 121(20), 10026-10040. doi: 10.1063/1.1798992

Available at: http://dx.doi.org/10.1063/1.1798992

This Article is brought to you for free and open access by the Chemistry at DigitalCommons@URI. It has been accepted for inclusion in Chemistry Faculty Publications by an authorized administrator of DigitalCommons@URI. For more information, please contact digitalcommons-group@uri.edu. 


\title{
Broken-symmetry unrestricted hybrid density functional calculations on nickel dimer and nickel hydride
}

\author{
Cristian V. Diaconu, ${ }^{\text {a) }}$ Art E. Cho, ${ }^{\text {b) }}$ and J. D. Doll \\ Department of Chemistry, Brown University, Providence, Rhode Island 02912 \\ David L. Freeman \\ Department of Chemistry, University of Rhode Island, Kingston, Rhode Island 02881
}

(Received 28 January 2004; accepted 4 August 2004)

\begin{abstract}
In the present work we investigate the adequacy of broken-symmetry unrestricted density functional theory for constructing the potential energy curve of nickel dimer and nickel hydride, as a model for larger bare and hydrogenated nickel cluster calculations. We use three hybrid functionals: the popular B3LYP, Becke's newest optimized functional Becke98, and the simple FSLYP functional (50\% Hartree-Fock and 50\% Slater exchange and LYP gradient-corrected correlation functional) with two basis sets: all-electron (AE) Wachters $+f$ basis set and Stuttgart RSC effective core potential (ECP) and basis set. We find that, overall, the best agreement with experiment, comparable to that of the high-level CASPT2, is obtained with B3LYP/AE, closely followed by Becke98/AE and Becke98/ECP. FSLYP/AE and B3LYP/ECP give slightly worse agreement with experiment, and FSLYP/ECP is the only method among the ones we studied that gives an unacceptably large error, underestimating the dissociation energy of $\mathrm{Ni}_{2}$ by $28 \%$, and being in the largest disagreement with the experiment and the other theoretical predictions. We also find that for $\mathrm{Ni}_{2}$, the spin projection for the broken-symmetry unrestricted singlet states changes the ordering of the states, but the splittings are less than $10 \mathrm{meV}$. All our calculations predict a $\delta \delta$-hole ground state for $\mathrm{Ni}_{2}$ and $\delta$-hole ground state for $\mathrm{NiH}$. Upon spin projection of the singlet state of $\mathrm{Ni}_{2}$, almost all of our calculations: Becke98 and FSLYP both AE and ECP and B3LYP/AE predict ${ }^{1}\left(d_{x^{2}-y^{2}}^{A} d_{x^{2}-y^{2}}^{B}\right)$ or ${ }^{1}\left(d_{x y}^{A} d_{x y}^{B}\right)$ ground state, which is a mixture of ${ }^{1} \Sigma_{g}^{+}$and ${ }^{1} \Gamma_{g}$. B3LYP/ECP predicts a ${ }^{3}\left(d_{x^{2}-y^{2}}^{A} d_{x y}^{B}\right)$ (mixture of ${ }^{3} \Sigma_{g}^{-}$and $\left.{ }^{3} \Gamma_{u}\right)$ ground state virtually degenerate with the ${ }^{1}\left(d_{x^{2}-y^{2}}^{A} d_{x^{2}-y^{2}}^{B}\right) /^{1}\left(d_{x y}^{A} d_{x y}^{B}\right)$ state. The doublet $\delta$-hole ground state of $\mathrm{NiH}$ predicted by all our calculations is in agreement with the experimentally predicted ${ }^{2} \Delta$ ground state. For $\mathrm{Ni}_{2}$, all our results are consistent with the experimentally predicted ground state of $0_{g}^{+}$(a mixture of ${ }^{1} \Sigma_{g}^{+}$and ${ }^{3} \Sigma_{g}^{-}$) or $0_{u}^{-}$(a mixture of ${ }^{1} \Sigma_{u}^{-}$and ${ }^{3} \Sigma_{u}^{+}$). (c) 2004 American Institute of Physics. [DOI: 10.1063/1.1798992]
\end{abstract}

\section{INTRODUCTION}

During the last decades clusters have been extensively studied because of their potential applications, their theoretical value in understanding the transition from isolated atomic systems to condensed matter ${ }^{1,2}$ and their relevance to the study of surface processes and heterogeneous catalysis. ${ }^{3-5}$ The rapid development of experimental techniques in recent years has made it possible both to obtain size-controlled transition metal clusters and to study their reactivity against chemisorption processes. ${ }^{6-9}$

Methods for studying properties and behavior of clusters have been developed, and a review on computational studies of clusters has been written by Freeman and Doll. ${ }^{10}$ There have been many studies on nickel clusters using various methods of exploring the potential energy surfaces (PES). The construction of such potential surfaces is a major problem, especially for transition metal clusters. Many methods have been used to construct PESs for nickel clusters, ranging

\footnotetext{
${ }^{a)}$ Electronic mail: cvdiaconu@brown.edu

b) Present address: Department of Chemistry and Center for Biomolecular Simulation, Columbia University, New York, NY 10027.
}

from empirical-Finis-Sinclair type, ${ }^{11,12}$ semiempiricaltight binding ${ }^{13,14}$ and extended Hückel, ${ }^{15}$ to ab initio or mixed empirical-ab initio ${ }^{16}$ approaches. Recently, there have been studies of hydrogen atoms on $\mathrm{Cu}$ surfaces ${ }^{17}$ within the density functional framework. It has been found that semiempirical methods are insufficient for accurate description of such systems, and first principle quantum-mechanical methods are needed to obtain a proper description of the hydrogen binding site.

Our long-term goal is to explore the structure and dynamics of clusters, including nickel and nickel hydride systems. The combination of the physical complexity and the computational demands of these systems necessitate that the microscopic force laws that are utilized in such simulations be both efficient and reliable.

Among the correlated electronic structure methods the best candidate is clearly density functional theory ${ }^{18,19}$ (DFT) because of its ability of reaching high accuracy-similar to coupled-cluster $\operatorname{CCSD}(\mathrm{T})$ method for second-row elements - when hybrid exchange-correlation functionals are used. ${ }^{20}$ Moreover, DFT (using hybrid functionals) is computationally not much more expensive than Hartree-Fock.

While there have been a number of DFT calculations 
reported on small nickel clusters, ${ }^{21-28}$ some results appear to be inconsistent both with respect to available experimental data and/or with respect to other theoretical predictions.

The works of Yanagisawa et al. ${ }^{29}$ and Barden et al. ${ }^{30}$ on the performance of DFT on the first transition metal series have shown that nonhybrid functionals (BLYP, ${ }^{31,32}$ BP86, ${ }^{31,33,34}$ BOP $^{31,35}$ and PW91 ${ }^{36}$ ) and hybrid functionals $\left(\mathrm{B} 3 \mathrm{LYP}^{32,37}, \mathrm{BHLYP}^{32,38}\right)$ give an overall similar description for $3 d$ transition metal dimers, with the nonhybrid ones giving better bond lengths and the hybrid ones better dissociation energies. However, while Yanagisawa et al. ${ }^{29}$ obtain good agreement with experiment for nickel dimer for all studied exchange-correlation functionals (they only calculated the triplet states), Barden et al. ${ }^{30}$ obtained a negative dissociation energy for their calculated singlet ground state with the B3LYP functional (and negative or very close to zero for all hybrid exchange-correlation functionals). This prompted us to use symmetry breaking in unrestricted DFT for describing the lowest singlet state of nickel dimer. With larger cluster calculations in mind, we also used broken symmetry unrestricted DFT to better describe bond breaking in all states of nickel dimer and nickel hydride.

It has been argued that broken-symmetry unrestricted calculations (Hartree-Fock and DFT with hybrid functionals) are useful for describing systems with weakly coupled electron pairs. ${ }^{20,39-44} \mathrm{Ni}_{2}$ is definitely such a case, as previously observed by Basch et al. ${ }^{21}$ As argued by Cremer, ${ }^{20}$ the combination of hybrid exchange-correlation functional with symmetry breaking leads to a better description of systems in which static correlation is present than does the restricted DFT formalism. Finally, we believe that the formalism used to describe any system is solely dictated by the objective of the calculation. For a variational approach, and DFT can be regarded as such-aside from the exchange-correlation functional-the more flexible is the form of the trial function (density), the lower is the obtained energy. Since our interest is mainly in the energetics of nickel clusters, the best choice for us seems to be the unrestricted broken symmetry DFT approach with hybrid functionals.

In the present work we study the nickel dimer and nickel hydride using broken symmetry unrestricted DFT with hybrid exchange-correlation functionals - mainly the popular B3LYP ${ }^{32,37}$ - as model systems for larger bare and hydrogenated nickel clusters in an attempt to establish what might comprise a minimally reliable method for more extensive nickel cluster calculations.

The outline of the remainder of the present paper is as follows: Section II discusses the used methods, Sec. III presents the results of the calculations, and, where possible, comparisons with previous reports. Section IV concludes with suggestions for further research based on the present findings.

\section{COMPUTATIONAL DETAILS}

The $\mathrm{DFT}^{18,19}$ calculations reported in this paper are carried out with NWCHEM $^{45}$ computational chemistry package, using the unrestricted Kohn-Sham ${ }^{19,46}$ approach, allowing for symmetry breaking, and using a finite orbital (spherical Gaussian) basis set expansion and charge density fitting.
Hartree-Fock and second order Møller-Plesset calculations are performed for comparison for the states of the $\mathrm{Ni}$ atom and are done in unrestricted form. ${ }^{47}$

Throughout the paper we will use the notation ${ }^{M}\left(h^{A} h^{B}\right)$ for the states of nickel dimer, where $M$ is the multiplicity, $h^{A}$ and $h^{B}$ are the unoccupied (hole) orbitals in the $3 d$ shell on the two $\mathrm{Ni}$ atoms, denoted $A$ and $B$. The broken symmetry singlet states (with $S_{z}=0$ and $\left\langle S^{2}\right\rangle=1$ ) are denoted by ${ }^{1,3}\left(h^{A} h^{B}\right)$.

In general, an unrestricted Slater or Kohn-Sham determinant is not an eigenfunction of the total spin operator $S^{2}$, and the results can only be characterized by the number of $\alpha$ and $\beta$ electrons. However, following common usage, we refer to the states that differ in the number of $\alpha$ and $\beta$ electrons by 0 as singlets, by 1 as doublets, and so on. We explicitly identify pure spin states where relevant.

\section{A. Exchange-correlation functionals}

We used three hybrid exchange-correlation (XC) functionals: the very popular B3LYP-composed of the B3, Becke's three-parameter hybrid exchange functional, ${ }^{37}$ and $\mathrm{LYP}^{32}$ correlation functional-is the first choice because it is well known and extensively characterized. Becke's newest optimized functional, Becke $98^{48}$ is also used, since it is supposed to be, in a certain sense, the best obtainable exchangecorrelation functional within the gradient-corrected framework. The hybrid composed of half Slater exchange, ${ }^{49}$ half Hartree-Fock exchange, and LYP ${ }^{32}$ correlation, named here FSLYP is also used for comparison, as it is the simplest theoretically justifiable hybrid method and is reported to perform rather well. ${ }^{42,43}$

\section{B. Basis sets}

All calculations are performed with spherical basis sets. As all-electron (AE) basis sets, Wachters $+f$ basis set, ${ }^{50-53}$ a $[14 s 11 p 6 d 3 f] /(8 s 6 p 4 d 1 f)$ contraction is used for nickel and $6-311++\mathrm{G}(2 d, 2 p)$, a $[6 s 2 p] /(4 s 2 p)$ contraction for hydrogen.

Effective-core potentials (ECP) are also explored, since they greatly reduce computational cost. Stuttgart RSC ECP effective core potentials basis set ${ }^{54,55}$ are used for nickel, as they provide a similar quality of valence basis functions as Wachters $+f$.

Ahlrichs Coulomb Fitting ${ }^{56,57}$ basis is used as a charge density (CD) fitting basis only for the all-electron calculations, as it significantly reduces computing time, especially for larger systems. When not specified otherwise, all reported all-electron results are obtained using charge density fitting.

We did not use charge density fitting with ECP because of the large errors that resulted when we tried the use of Ahlrichs Coulomb Fitting basis in combination with Stuttgart RSC ECP. For example, for B3LYP functional, CD fitting error is as much as $0.3 \mathrm{eV}$ for both the interconfigurational energies of $\mathrm{Ni}$ atom and the binding energy of $\mathrm{Ni}_{2}$. Please refer to Appendix A for discussion of the accuracy of charge density fitting. 


\section{Numerical integration and convergence}

The numerical integration necessary for the evaluation of the exchange-correlation energy implemented in NWCHEM uses an Euler-MacLaurin scheme for the radial components (with a modified Mura-Knowles transformation) and a Lebedev scheme for the angular components. We use three levels of accuracy for the numerical integration that are used in our DFT calculations, labeled by the corresponding keywords from NWCHEM (medium, fine, and xfine).

The reported atomic calculations are those obtained with the xfine grid. For geometry optimization and vibrational frequency calculations we use the fine grid. And for the potential energy curve (PEC) scans we used the medium grid. The maximum number of iterations is set to 100 in all calculations.

Please refer to Appendix B for details on numerical integration and convergence criteria.

\section{Initial guess}

For all DFT methods we first performed a calculation for $\mathrm{Ni}$ atom using fractional occupation numbers (FONs), ${ }^{58}$ as implemented in NWCHEM. We use an exponent of 0.01 hartree for the Gaussian broadening function. We then use the molecular orbitals from the FONs calculation, after proper reordering, as initial guess for computing the ${ }^{3} F$ and ${ }^{3} D$ states of Ni atom. We use $t_{2 g}^{6} e_{g}^{2}$ configuration in the $O_{h}$ symmetry group for the ${ }^{3} F$ state. In order to obtain the lowest energy possible for the ${ }^{3} D$ state, we scan all hole positions: $d_{z^{2}}, d_{x^{2}-y^{2}}$, and $d_{x y}$ using $D_{4 h}$ symmetry group, and $d_{x z}$ and $d_{y z}$ using $D_{2 h}$ symmetry group, enforcing the position of the hole with a maximum overlap condition.

For $\mathrm{Ni}_{2}$ and $\mathrm{NiH}$, we use a broken-symmetry initial guess of the form: $3 d^{9} 4 s^{1} \uparrow \uparrow+\downarrow \downarrow 3 d^{9} 4 s^{1}$ for singlet $\mathrm{Ni}_{2}$, $3 d^{9} 4 s^{1} \uparrow \uparrow+\downarrow \uparrow 3 d^{9} 4 s^{1}$ for triplet $\mathrm{Ni}_{2}$ and $\mathrm{Ni} 3 d^{9} 4 s^{1} \uparrow \uparrow$ $+\downarrow 1 s^{1} \mathrm{H}$ for $\mathrm{NiH}$. As initial guess molecular orbitals we use those from the $\mathrm{Ni}$ atom calculations, sweeping through all unique positions of the holes in the $3 d$ orbitals of $\mathrm{Ni}$ atom(s), and enforcing the position of the hole(s) with a maximum overlap condition.

\section{E. Geometry optimization}

Geometry optimizations are performed using the DRIVER module of NWCHEM using NWCHEM's default convergence criteria (in atomic units): $4.5 \times 10^{-4}$ maximum and $3.0 \times 10^{-4}$ root mean square gradient, $1.8 \times 10^{-3}$ maximum and $1.2 \times 10^{-3}$ root mean square of the cartesian step. These convergence criteria give a maximum error in equilibrium bond length of less than $\approx 10^{-3} \AA$ for $\mathrm{Ni}_{2}$ and less than $\approx 5 \times 10^{-4} \AA$ for $\mathrm{NiH}$. The available precision is set to 5 $\times 10^{-7}$ hartree for the fine grid and $5 \times 10^{-8}$ hartree for the xfine grid.

\section{F. Vibrational frequencies}

Harmonic vibrational frequencies are calculated using NWCHEM's VIB module with the default options. Since analytical Hessian for open shell systems is not available for the exchange-correlation functionals used, the Hessian is com- puted by finite differences with $\Delta=0.01 \mathrm{bohr}$, which gives an estimated error for the vibrational frequencies of $\approx 0.5 \mathrm{~cm}^{-1}(\approx 0.25 \%)$ for $\mathrm{Ni}_{2}$ and $\approx 2 \mathrm{~cm}^{-1}(\approx 0.1 \%)$ for $\mathrm{NiH}$.

\section{G. Spin and symmetry projection}

In general, an open-shell Slater or Kohn-Sham determinant is not an eigenfunction of the total spin operator $S^{2}$. However, spin-adapted configurations can be obtained as combinations of (a small number of) restricted determinants. ${ }^{59,60}$ Unrestricted determinants are not eigenfunction of the total spin operator $S^{2}$, either, and they cannot be spin-adapted by combining a small number of unrestricted determinants. ${ }^{59}$ However, for antiferromagnetic coupling of two weakly interacting identical high spin monomers, Noodleman ${ }^{40}$ derived an approximate spin projection scheme that is correct to the first order in the overlap integrals. $\mathrm{Ni}_{2}$ can be well approximated by such a model.

As previously observed by Basch et al. ${ }^{21}$ the electronic structure of nickel clusters corresponds roughly to a model in which the $3 d$ electrons can be viewed as weakly interacting localized $3 d^{9}$ units bound together primarily by $4 s$ electrons. If the $4 s$ electrons are paired in a $\sigma$ bond, then $\mathrm{Ni}_{2}$ has two possible spin states: singlet and triplet. However, the openshell singlet state cannot be represented by a single determinant, and the broken-symmetry single determinant $\Psi_{B}$ obtained by putting one of the open-shell electrons in a spin $\alpha$ $d$ orbital on one of the Ni atoms and the other electron in a spin $\beta d$ orbital on the other Ni atom is not pure singlet, but an equal mixture of singlet and triplet (using $\left|S, S_{z}\right\rangle$ notation for the spin states):

$$
\Psi_{B}=\frac{1}{\sqrt{2}}|0,0\rangle+\frac{1}{\sqrt{2}}|1,0\rangle
$$

with the expectation value of the total spin $\left\langle\Psi_{B}\left|S^{2}\right| \Psi_{B}\right\rangle$ $=1$. In agreement with this model, for the broken-symmetry calculations of the $S_{z}=0$ state of the Ni dimer the expectation value of the total spin $\left\langle S^{2}\right\rangle$ is close to the exact value of 1 for the broken-symmetry mixed state, and for the triplet $\left(S_{z}=1\right)$ state, $\left\langle S^{2}\right\rangle$ is close to the exact value of 2 (in both cases, the relative absolute differences between the computed and the exact values are less than $2 \%$ ). Mulliken population analysis also supports the weakly interacting $3 d^{9}$ units model. For the triplet nickel dimer there is a Mulliken spin population of 1.00 on each $\mathrm{Ni}$ atom, and for the brokensymmetry singlet there is a Mulliken spin population of 1.1 on one of the $\mathrm{Ni}$ atoms and -1.1 on the other.

Using the approximate projection method of Noodleman, ${ }^{40}$ the energy of the pure singlet state, $E(0)$ can be obtained from the energy of the unrestricted brokensymmetry singlet $E_{B}$, and the energy of the triplet $E(1)$ :

$$
E(0)=2 E_{B}-E(1) \text {. }
$$

The same result can be also obtained by the spin projection technique (see, e.g., Refs. 61 and 62).

$\mathrm{Ni}_{2}$ belongs to $D_{\infty h}$ point symmetry group, and the irreducible representations (irreps.) are good quantum numbers for the molecular states. We combine the spin projection with 
TABLE I. Energies of atomic states of Ni. Values are in $\mathrm{eV}$, relative to the ground state.

\begin{tabular}{|c|c|c|c|c|c|c|c|c|c|c|c|}
\hline \multirow[b]{2}{*}{ State } & \multirow[b]{2}{*}{ Expt. $^{a}$} & \multirow[b]{2}{*}{$\mathrm{RC}^{\mathrm{b}}$} & \multirow[b]{2}{*}{ Expt. $-\mathrm{RC}^{\mathrm{c}}$} & \multirow{2}{*}{$\begin{array}{c}\mathrm{UHF} \\
\mathrm{AE}\end{array}$} & \multirow{2}{*}{$\begin{array}{c}\text { MP2 } \\
\text { AE }\end{array}$} & \multicolumn{2}{|c|}{ FSLYP } & \multicolumn{2}{|c|}{ B3LYP } & \multicolumn{2}{|c|}{ Becke98 } \\
\hline & & & & & & $\mathrm{AE}$ & $\mathrm{ECP}$ & $\mathrm{AE}$ & ECP & $\mathrm{AE}$ & ECP \\
\hline${ }^{3} D\left(3 d^{9} 4 s^{1}\right)$ & 0 & & 0 & 1.44 & 0.27 & 0.12 & 0.32 & 0 & 0.01 & 0 & 0.20 \\
\hline${ }^{3} F\left(3 d^{8} 4 s^{2}\right)$ & 0.03 & -0.36 & 0.39 & 0 & 1.41 & 0 & 0 & 0.36 & 0 & 0.29 & 0 \\
\hline${ }^{1} S\left(3 d^{10}\right)$ & 1.74 & 0.21 & 1.53 & 5.81 & 0 & 2.62 & 3.02 & 1.90 & 2.21 & 1.78 & 2.37 \\
\hline
\end{tabular}

${ }^{a}$ Weighted averages over the $J$ components of the experimental values (Ref. 64).

${ }^{\mathrm{b}}$ Martin and Hay estimations of relativistic corrections from Ref. 65.

${ }^{\mathrm{c}}$ Experimental values with relativistic corrections subtracted.

symmetry projection to extract the maximum information possible from the single-determinant Kohn-Sham DFT calculations. From simple group-theoretical considerations one can find that the pure spin and symmetry states of $\mathrm{Ni}_{2}$ that arise from $d_{\delta}$ orbitals, which are found to give the lowest energy states for all calculations, are: ${ }^{1} \Sigma_{g}^{+},{ }^{1} \Gamma_{g},{ }^{1} \Sigma_{u}^{-}$, ${ }^{3} \Sigma_{g}^{-},{ }^{3} \Sigma_{u}^{+}$, and ${ }^{3} \Gamma_{u}$. Within the model of two weakly interacting $3 d^{9}$ units, for the purpose of projection we consider only the active electrons and the active orbitals on each center, namely, $d_{x^{2}-y^{2}}^{A}, d_{x y}^{A}, d_{x^{2}-y^{2}}^{B}$, and $d_{x y}^{B}$.

The projection has been carried out using the projection operators technique in $D_{8 h}$, which the smallest subgroup of $D_{\infty h}$ in which all irreps. arising from the $\left(d_{\delta}^{A}\right)^{1}\left(d_{\delta}^{B}\right)^{1}$ configuration can be completely correlated, and the following equations relating the energies of the pure spin and symmetry states listed above to the energies of the computed triplet and projected singlet states are obtained:

$$
\begin{aligned}
& E\left[{ }^{1}\left(d_{x^{2}-y^{2}}^{A} d_{x^{2}-y^{2}}^{B}\right)\right]=\frac{1}{2}\left[E\left({ }^{1} \Sigma_{g}^{+}\right)+E\left({ }^{1} \Gamma_{g}\right)\right], \\
& E\left[{ }^{3}\left(d_{x^{2}-y^{2}}^{A} d_{x^{2}-y^{2}}^{B}\right)\right]=\frac{1}{2}\left[E\left({ }^{3} \Sigma_{u}^{+}\right)+E\left({ }^{3} \Gamma_{u}\right)\right], \\
& E\left[{ }^{1}\left(d_{x y}^{A} d_{x y}^{B}\right)\right]=\frac{1}{2}\left[E\left({ }^{1} \Sigma_{g}^{+}\right)+E\left({ }^{1} \Gamma_{g}\right)\right], \\
& E\left[{ }^{3}\left(d_{x y}^{A} d_{x y}^{B}\right)\right]=\frac{1}{2}\left[E\left({ }^{3} \Sigma_{u}^{+}\right)+E\left({ }^{3} \Gamma_{u}\right)\right], \\
& E\left[{ }^{1}\left(d_{x^{2}-y^{2}}^{A} d_{x y}^{B}\right)\right]=\frac{1}{2}\left[E\left({ }^{1} \Sigma_{u}^{-}\right)+E\left({ }^{1} \Gamma_{g}\right)\right], \\
& E\left[{ }^{3}\left(d_{x^{2}-y^{2}}^{A} d_{x y}^{B}\right)\right]=\frac{1}{2}\left[E\left({ }^{3} \Sigma_{g}^{-}\right)+E\left({ }^{3} \Gamma_{u}\right)\right] .
\end{aligned}
$$

These equations contain the maximal information that can be obtained from single-determinant calculations.

From Eq. (2) we can derive the (partially) symmetry adapted equivalent of Eq. (1):

$$
E\left[{ }^{1}\left(h^{A} h^{B}\right)\right]=2 E\left[{ }^{1,3}\left(h^{A} h^{B}\right)\right]-E\left[{ }^{3}\left(h^{A} h^{B}\right)\right],
$$

where $\left(h^{A} h^{B}\right)$ represents each of $\left(d_{x^{2}-y^{2}}^{A} d_{x^{2}-y^{2}}^{B}\right)$, $\left(d_{x^{2}-y^{2}}^{A} d_{x y}^{B}\right)$, and $\left(d_{x y}^{A} d_{x y}^{B}\right)$. The spin projection has to be done separately for each of the combinations of holes $\left(d_{x^{2}-y^{2}}^{A} d_{x^{2}-y^{2}}^{B}\right)$ and $\left(d_{x^{2}-y^{2}}^{A} d_{x y}^{B}\right)$.

Since the equations for the states ${ }^{M}\left(d_{x y}^{A} d_{x y}^{B}\right)$ have a similar form to those for the ${ }^{M}\left(d_{x^{2}-y^{2}}^{A} d_{x^{2}-y^{2}}^{B}\right)$ states, ${ }^{M}\left(d_{x^{2}-y^{2}}^{A} d_{x^{2}-y^{2}}^{B}\right)$ and ${ }^{M}\left(d_{x y}^{A} d_{x y}^{B}\right)$ states should have the same energy $[M$ can be 1,3 or $(1,3)]$. We calculate the ${ }^{1,3}\left(d_{x y}^{A} d_{x y}^{B}\right)$ and ${ }^{3}\left(d_{x y}^{A} d_{x y}^{B}\right)$ states for consistency check.
Since the bond lengths for the pure spin states are different from each other and from the mixed state, we use a harmonic approximation of the potential around equilibrium bond length for each state:

$$
E(d)=-D_{e}+\frac{1}{2} \mu \omega_{e}^{2}\left(d-d_{e}\right)^{2}
$$

and solve the resulting equations for $d_{e}$ (equilibrium bond lenth), $D_{e}$ (dissociation energy), and $\omega_{e}$ (vibrational frequency) for the projected state (here $\mu$ denotes the reduced mass of the molecule).

\section{RESULTS AND DISCUSSIONS}

\section{A. Nickel atom}

The ground state of the nickel atom is ${ }^{3} F_{4}\left(3 d^{8} 4 s^{2}\right) .{ }^{63,64}$ However, since our calculations do not include spin-orbit coupling, we use weighted averages over the $J$ components of the experimental data for comparison, which makes ${ }^{3} D\left(3 d^{9} 4 s^{1}\right)$ the ground state, with ${ }^{3} F\left(3 d^{8} 4 s^{2}\right)$ state only $0.03 \mathrm{eV}$ higher, and ${ }^{1} S\left(3 d^{10}\right)$ state $1.74 \mathrm{eV}$ above the ground state.

As first estimated by Martin and Hay ${ }^{65}$ and confirmed by full relativistic calculations done by Jeng and Hsue ${ }^{66}$ the relativistic effects in the $3 d$ transition metal series are important. Therefore, in comparing our nonrelativistic calculations with the experiment we take such effects into account by subtracting the estimated values reported by Martin and Hay from the experimental values. After this correction (see Table I for details), the ground state remains ${ }^{3} \mathrm{D}$, with ${ }^{3} F$ state 0.39 $\mathrm{eV}$ higher, and ${ }^{1} S$ state $1.53 \mathrm{eV}$ above the ground state. These values will be referred to as "relativistically corrected (RC) experimental values."

In Table I we choose to utilize the Martin and Hay ${ }^{65}$ relativistic corrections as opposed to the ones computed by Jeng and Hsue ${ }^{66}$ because they include the additional ${ }^{1} S\left(3 d^{10}\right)$ configuration. The results of the recent relativistic calculations in the RESC approximation (relativistic scheme by eliminating small components) reported by Yanagisawa et $a .^{29}$ do not lend themselves to an analysis of relativistic corrections. Moreover, these calculations seem to be at odds with the two previous calculations.

Our results, summarized in Table I, show that only the DFT/Wachters $+f$ calculations with B3LYP and Becke98 hybrid exchange-correlation functionals predict a ${ }^{3} D$ ground state, although B3LYP/ECP predicts the ${ }^{3} D$ state only 0.01 $\mathrm{eV}$ above the ${ }^{3} \mathrm{~F}$ ground state. 
TABLE II. Ground state of $\mathrm{Ni}_{2}$ —comparison between computations and experiment. The reported singlet states from our calculations are projected. $d_{e}$, bond length $(\AA) ; D_{e}$, dissociation energy, relative to ground state $\mathrm{Ni}$ atoms (without zero-point correction, $\mathrm{eV}) ; \omega_{e}$, vibrational frequency $\left(\mathrm{cm}^{-1}\right)$. The relative deviations from the experimental values are given in parentheses, and the average (AARD) and maximum (MARD) absolute relative deviations from experimental values of $d_{e}, D_{e}$, and $\omega_{e}$ are listed under AARD and MARD columns, respectively.

\begin{tabular}{|c|c|c|c|c|c|c|}
\hline Method & State & $d_{e}$ & $D_{e}$ & $\omega_{e}$ & AARD & MARD \\
\hline FSLYP/ECP & ${ }^{1}\left(d_{x y}^{A} d_{x y}^{B}\right)$ & $2.236(1.5)$ & $1.325(-28.4)$ & $283.0(14.9)$ & 14.9 & 28.4 \\
\hline FSLYP/AE & ${ }^{1}\left(d_{x y}^{A} d_{x y}^{B}\right)$ & $2.260(2.5)$ & $1.664(-10.1)$ & $271.1(10.1)$ & 7.6 & 10.1 \\
\hline Becke98/AE & ${ }^{1}\left(d_{x y}^{A} d_{x y}^{B}\right)$ & $2.296(4.2)$ & $2.071(11.9)$ & $256.8(4.3)$ & 6.8 & 11.9 \\
\hline CASPT2 $^{\mathrm{a}}$ & ${ }^{1} \Sigma_{g}^{+},{ }^{1} \Gamma_{g}$ & $2.281(3.5)$ & $1.89(2.2)$ & $281.0(14.1)$ & 6.6 & 14.1 \\
\hline CASSCF/IC-ACPF ${ }^{b}$ & ${ }^{1} \Gamma_{g}^{g}$ & $2.291(3.9)$ & $1.691(-8.6)$ & $253.0(2.8)$ & 5.1 & 8.6 \\
\hline Becke98/ECP & ${ }^{1}\left(d_{x^{2}-y^{2}}^{A} d_{x^{2}-y^{2}}^{B}\right)$ & $2.278(3.4)$ & $1.792(-3.1)$ & $265.1(7.7)$ & 4.7 & 7.7 \\
\hline B3LYP/ECP & ${ }^{3}\left(d_{x^{2}-y^{2}}^{A} d_{x y}^{B}\right)$ & $2.271(3.0)$ & $1.851(0.1)$ & $269.3(9.4)$ & 4.2 & 9.4 \\
\hline B3LYP/AE & ${ }^{1}\left(d_{x^{2}-y^{2}}^{A} d_{x^{2}-y^{2}}^{B}\right)$ & $2.291(3.9)$ & $1.835(-0.8)$ & $258.9(5.2)$ & 3.3 & 5.2 \\
\hline Expt. $^{c}$ & $0_{g}^{+} / 0_{u}^{-}$ & 2.204 & 1.85 & 246.2 & & \\
\hline
\end{tabular}

${ }^{\mathrm{a}}$ We report here the values from Table VIII of Ref. 67, last column $\left(+3 s 3 p\right.$ for $d_{e}$ and $\omega_{e}$, and BSSE for $\left.D_{e}\right)$, from which we subtract the estimated relativistic corrections (RC) and, for $D_{e}$ only, the estimated spin-orbit coupling contributions (SO). From the same table we estimate the relativistic corrections to $d_{e}, D_{e}$, and $\omega_{e}$ as the difference between the values in the $+\mathrm{RC}$ column and ones in the CASSCF column, and the spin-orbit coupling contribution to $D_{e}$ as the difference between the value in the + SO column and the one in the $+3 s 3 p$ column. We also subtract these RC and SO contributions from the experimental values.

${ }^{\mathrm{b}}$ From Ref. 72.

${ }^{\mathrm{c}}$ Experimental values from which we subtract the CASPT2 estimates (see footnote a) from Ref. 67 for (RC) for $d_{e}, D_{e}$, and $\omega_{e}$, and SO contributions for $D_{e}$. The experimental value of $d_{e}$ is $2.1545 \pm 0.0004 \AA$ (Ref. 68) from which we subtract the CASPT2 RC of $-0.05 \AA$. The experimental value of $D_{0}$ is $2.042 \pm 0.002 \mathrm{eV}$ (Ref. 68), from which we subtract the CASPT2 RC of $0.07 \mathrm{eV}$ and CASPT2 SO of $0.14 \mathrm{eV}$; we report $D_{e}=D_{0}$ $+\frac{1}{2} \hbar \omega_{e}$. The experimental value of $\omega_{e}$ is $259.2 \pm 3.0 \mathrm{~cm}^{-1}$ (Ref. 70) from which we subtract the CASPT2 RC of $13 \mathrm{~cm}^{-1}$. An earlier work ${ }^{71}$ reported $280 \pm 20 \mathrm{~cm}^{-1}$.

It is worth mentioning that, for all our DFT calculations, there are differences between the components of the ${ }^{3} D$ state of $\mathrm{Ni}$ and these differences range from $4 \mathrm{meV}$ to $37 \mathrm{meV}$. We report the energy of the ${ }^{3} D$ component with the lowest energy as the energy of the ${ }^{3} D$ state. It is also worth mentioning that the B3LYP/ECP calculations fail to converge for the spin $\alpha d_{x y^{-}}, d_{y z^{-}}, d_{x z^{-}}$, and $d_{x^{2}-y^{2}}$-hole components of the ${ }^{3} D$ state.

The all-electron calculations with B3LYP and Becke98 $\mathrm{XC}$ functionals also predict an ordering of the ${ }^{3} D,{ }^{3} F$, and ${ }^{1} S$ states in agreement with the experiment.

The values of the computed energies of ${ }^{3} F$ (relative to ${ }^{3} D$ ) differ from the observed experimental values by $0.30 \mathrm{eV}$ (B3LYP) and $0.26 \mathrm{eV}$ (Becke98). However, when compared with the relativistically corrected experimental values, the differences drop to only $-0.06 \mathrm{eV}$ and $-0.10 \mathrm{eV}$, respectively. On the other hand, the computed energies of ${ }^{1} S$ (relative to ${ }^{3} D$ ) are larger than the observed experimental values by $0.16 \mathrm{eV}$ (B3LYP) and $0.04 \mathrm{eV}$ (Becke98), and larger than the relativistically corrected experimental values by $0.37 \mathrm{eV}$ and $0.25 \mathrm{eV}$, respectively. However, the larger errors in the ${ }^{1} S$ is less important for the purpose of nickel cluster calculations.

Hartree-Fock calculations predict ${ }^{3} F$ ground state, ${ }^{3} D$ $1.44 \mathrm{eV}$ higher and ${ }^{1} S 5.81 \mathrm{eV}$ above the ground state in good agreement with numerical HF calculations of Martin and Hay, ${ }^{65}$ but with large errors compared to the RC experimental values. MP2 calculations predict ${ }^{1} S$ ground state, with ${ }^{3} D$ and ${ }^{3} F$ states $0.27 \mathrm{eV}$ and $1.41 \mathrm{eV}$ higher, respectively.

The unoptimized FSLYP functional is, as expected, the least accurate. With the Wachters $+f$ basis it yields results that differ from the RC experimental values and B3LYP and Becke98 results by $\approx-0.5 \mathrm{eV}$ for ${ }^{3} F$ and by $\approx 0.5 \mathrm{eV}$ for ${ }^{1} S$.

The ECPs tend to overstabilize ${ }^{3} F$ by $0.2-0.5 \mathrm{eV}$ and destabilize ${ }^{1} S$ by $0.2-0.4 \mathrm{eV}$ (relative to ${ }^{3} D$ ) with respect to the all-electron counterparts. Thus, all our DFT/ECP calculations predict ${ }^{3} F$ ground state. However, the B3LYP/ECP calculations yield ${ }^{3} D$ only $0.01 \mathrm{eV}$ above the ${ }^{3} F$ ground state, which can be considered acceptable error for the dissociation energy of nickel dimer which is of order of $2 \mathrm{eV}$, given the savings of using ECPs.

\section{B. Nickel dimer}

The determination of the ground state of $\mathrm{Ni}_{2}$ has been debated over the last few decades. According to the recent results, ${ }^{67,68}$ the most plausible candidates are spin-orbit coupled states of $\Omega=0_{g}^{+}$(a mixture of ${ }^{3} \Sigma_{g}^{-}$and ${ }^{1} \Sigma_{g}^{+}$) and $\Omega=0_{u}^{-}\left(\right.$a mixture of ${ }^{3} \Sigma_{u}^{+}$and $\left.{ }^{1} \Sigma_{u}^{-}\right)$.

The bond lengths $\left(d_{e}\right)$, dissociation energies $\left(D_{e}\right)$ (Ref. 69) and vibrational frequencies $\left(\omega_{e}\right)$ for the ground state of $\mathrm{Ni}_{2}$ from different calculations are reported in Table II along with experimental values and results from other theoretical studies. The results in Table II are listed in the order of decreasing average absolute relative deviations (AARD) from experimental values of bond length $\left(d_{e}\right)$, dissociation energy $\left(D_{e}\right)$ and vibrational frequency $\left(\omega_{e}\right)$.

Please note that our calculations are nonrelativistic and do not include spin-orbit coupling, and spin-orbit deperturbed values of molecular properties of interest for $\mathrm{Ni}_{2}$ are 
not available in the literature. To account for that, we have subtracted the CASPT2 RC to $d_{e}, D_{e}$, and $\omega_{e}$, and spinorbit contributions (SO) to $D_{e}$ from the experimental values. ${ }^{68,70,71}$ We estimate the relativistic and spin-orbit coupling corrections from Ref. 67. Please see footnote a of Table II for details.

The reported singlet states from our calculations are spin projected by the approximate method described in Sec. II (Computational details).

For the results from FSLYP/ECP and Becke98/ECP computations, the splitting between the $\left(d_{x y}^{A} d_{x y}^{B}\right)$ and $\left(d_{x^{2}-y^{2}}^{A} d_{x^{2}-y^{2}}^{B}\right)$ states, both for triplet and for mixed $S_{z}=0$, is larger ( $8 \mathrm{meV}$ for FSLYP/ECP and $4 \mathrm{meV}$ for Becke98/ECP) than the accuracy of the DFT calculations (better than 0.1 $\mathrm{meV}$ ). Thus, our approximate spin and symmetry projections are questionable for these particular calculations. However, since we observed even larger differences between the components of the ${ }^{3} \mathrm{D}$ state of $\mathrm{Ni}$ (up to $0.03 \mathrm{eV}$ ), we chose not to investigate this matter any further. In these cases, the reported values are those of the component with the lowest total energy (largest dissociation energy).

Almost all of our calculations: Becke98 and FSLYP both $\mathrm{AE}$ and ECP and B3LYP/AE predict ${ }^{1}\left(d_{x^{2}-y^{2}}^{A} d_{x^{2}-y^{2}}^{B}\right)$ or ${ }^{1}\left(d_{x y}^{A} d_{x y}^{B}\right)$ ground state, which is a mixture of ${ }^{1} \sum_{g}^{+}$and ${ }^{1} \Gamma_{g}$. B3LYP/ECP predicts a ${ }^{3}\left(d_{x^{2}-y^{2}}^{A} d_{x y}^{B}\right)$ (mixture of ${ }^{3} \Sigma_{g}^{-}$and $\left.{ }^{3} \Gamma_{u}\right)$ ground state virtually degenerate with the ${ }^{1}\left(d_{x^{2}-y^{2}}^{A} d_{x^{2}-y^{2}}^{B}\right) /{ }^{1}\left(d_{x y}^{A} d_{x y}^{B}\right)$ state, which is only $1 \mathrm{meV}$ higher in energy than ${ }^{3}\left(d_{x^{2}-y^{2}}^{A} d_{x y}^{B}\right)$ ground state.

Among the high-level wave function methods, CASPT $2{ }^{67}$ without spin-orbit coupling predicts ${ }^{1} \Sigma_{g}^{+}$ground state degenerate with ${ }^{1} \Gamma_{g}$, and CASSCF/IC-ACPF ${ }^{72}$ predicts ${ }^{1} \Gamma_{g}$ ground state. Our DFT all-electron calculations can be consistent with either one of the wave function methods. The experimental results are consistent with any of the predictions of our DFT calculations and CASPT2 $2{ }^{67}$ but not with the ${ }^{1} \Gamma_{g}$ state predicted by CASSCF/IC-ACPF. ${ }^{72}$

The absolute relative deviations from the experimental values of computed bond lengths $d_{e}$, dissociation energies $D_{e}$, and vibrational frequencies $\omega_{e}$ for $\mathrm{Ni}_{2}$ are plotted in Fig. 1 , arranged from left to right in order of decreasing total absolute relative deviation (TARD) - the sum of absolute relative deviations from the experimental values of the computed $d_{e}, D_{e}$ and $\omega_{e}$.

From Fig. 1, as well as from Table II, it is apparent that overall, for $\mathrm{Ni}_{2}$ the all-electron DFT calculations with B3LYP functional give the best agreement with experiment (9.9\% TARD). B3LYP/ECP (12.5\% TARD) and Becke98/ ECP (14.2\% TARD) follow with an overall performance just a little better than CASSCF/IC-ACPF ${ }^{72}$ (15.3\% TARD). Becke98/AE (20.4\% TARD) and FSLYP/AE (22.7\% TARD) are next among our DFT calculations, performing just a few percent worse than CASPT2 ${ }^{67}$ (19.8\% TARD). With $44.8 \%$ TARD, the FSLYP/ECP calculation gives the largest disagreement with experiment and the other methods.

The relative deviations from the experimental values of the computed bond length $\left(d_{e}\right)$, dissociation energy $D_{e}$, asymptotic dissociation energy $D_{e}^{a}$, (vide infra), and vibrational frequency $\omega_{e}$ of $\mathrm{Ni}_{2}$ are plotted in Fig. 2 for compari-

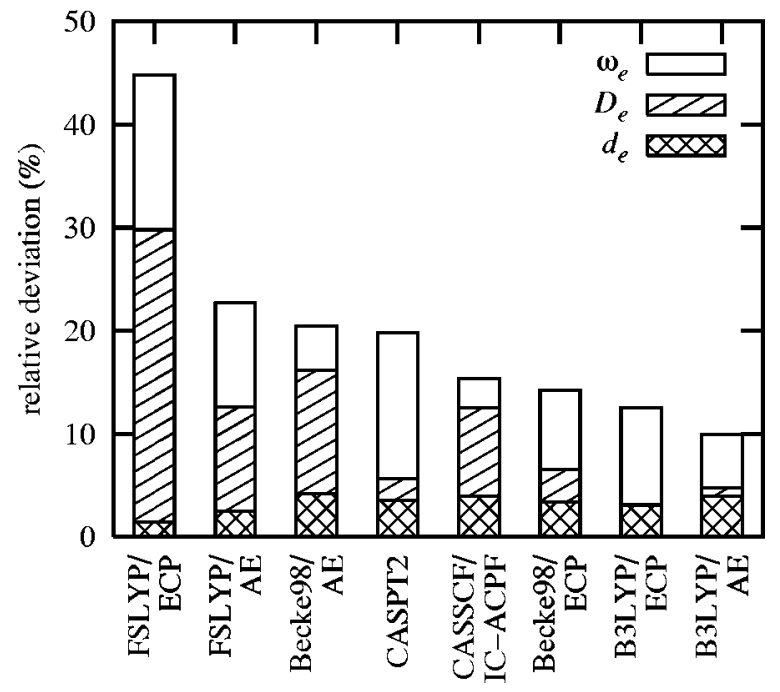

FIG. 1. The absolute relative deviations from experiment of computed dissociation energy, bond length, and vibrational frequency for $\mathrm{Ni}_{2}$. The results are arranged from left to right in order of decreasing total absolute relative deviation (TARD) - the sum of absolute relative deviations from the experimental values of the computed $d_{e}, D_{e}$, and $\omega_{e}$.

son. The values are arranged in order of increasing deviation in the bond length.

\section{Bond length}

It is apparent that all calculations included in Table II and Fig. 2-both our DFT calculations and the CASPT2 ${ }^{67}$ and CASSCF/IAACPF ${ }^{72}$ wave function methods included for comparison-overestimate the bond length of $\mathrm{Ni}_{2}$. The deviations from the experimental value of the computed bond length, $\Delta d_{e}=d_{e}^{\text {comp }}-d_{e}^{\text {expt }}$ range between $0.03 \AA$ $(1.5 \%)$ and $0.09 \AA(4.2 \%)$.

Among our DFT calculations, the best agreement with the experiment for the bond length of $\mathrm{Ni}_{2}$ is obtained by FSLYP/ECP with $\Delta d_{e}=0.032 \AA(1.5 \%)$, followed by

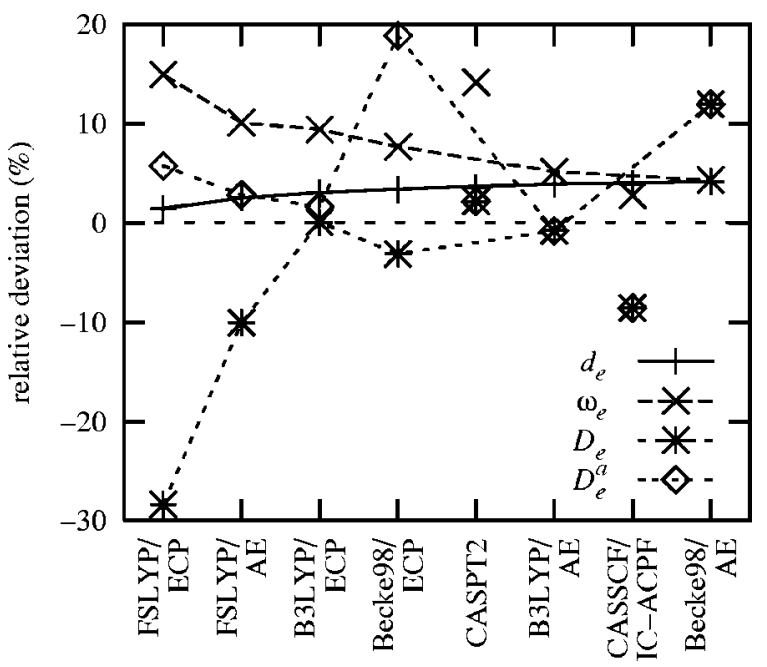

FIG. 2. The relative deviations from experimental values of the computed bond length $d_{e}$, dissociation energy $D_{e}$, asymptotic dissociation energy ( $D_{e}^{a}$, see text for definition), and vibrational frequency $\omega_{e}$ of $\mathrm{Ni}_{2}$. Only the results from our DFT calculations are connected by lines. CASPT2 and $\mathrm{CASSCF} / \mathrm{IC}-\mathrm{ACPF}$ are included for comparison. 
FSLYP/AE with $\Delta d_{e}=0.056 \AA(2.5 \%)$ and B3LYP/ECP with $\Delta d_{e}=0.067 \AA$ (3.0\%). Becke98/ECP with $\Delta d_{e}$ $=0.074 \AA(3.4 \%)$ performs very similar to CASPT2, for which $\Delta d_{e}=0.077 \AA$ (3.5\%). Both B3LYP/AE and CASSCF/IC-ACPF are among the methods that give the largest disagreement with the experiment, with $\Delta d_{e}$ $=0.087 \AA(3.9 \%)$. Finally, Becke98/AE yields the worst deviation from experiment, $\Delta d_{e}=0.092 \AA(4.2 \%)$.

Among all three XC functionals, the best agreement with experiment for the bond length is obtained with the FSLYP functional, both AE and ECP. B3LYP follows with a bond length $0.03 \AA$ longer than the one computed with FSLYP. Becke98 bond length is in the worst agreement with the experiment, but only $\approx 0.005 \AA$ longer than the B3LYP bond length.

For each of the three XC functionals used, ECP calculation predicts shorter bond length than the $\mathrm{AE}$ one by $\approx 0.02 \AA$, and, thus, it is in better agreement with the experiment.

\section{Dissociation energy}

The computed dissociation energies span a large range of values, from $1.33 \mathrm{eV}$ for FSLYP/ECP to $2.07 \mathrm{eV}$ for Becke98/AE. The deviations from the experimental value of the computed dissociation energy, $\Delta D_{e}=D_{e}^{\text {comp }}-D_{e}^{\text {expt }}$ range between $-0.525 \mathrm{eV}(-28.4 \%)$ and $0.221 \mathrm{eV}(11.9 \%)$.

Among our DFT calculations, the best agreement with the experiment for the dissociation energy of $\mathrm{Ni}_{2}$ is obtained with the B3LYP functional. B3LYP/ECP slightly overestimates $D_{e}$ by $0.001 \mathrm{eV}(0.1 \%)$, while B3LYP/AE slightly underestimates $D_{e}$ by $0.015 \mathrm{eV}(0.8 \%)$. This excellent agreement with the experiment of the B3LYP functional is clearly fortuitous since the errors in the B3LYP dissociation energies average $0.10 \mathrm{eV}$, with a maximum absolute deviation of 0.36 $\mathrm{eV}$ for the G2 set of molecules. ${ }^{73}$ Becke98/ECP comes second and underestimates $D_{e}$ by $0.058 \mathrm{eV}(3.1 \%)$, performing only slightly worse than CASPT2, which overestimates $D_{e}$ by $0.040 \mathrm{eV}(2.2 \%)$. FSLYP/AE is next and it underestimates $D_{e}$ by $0.186 \mathrm{eV}(10.1 \%)$ similar to CASSCF/IC-ACPF, for which $\Delta D_{e}=-0.159 \mathrm{eV}(-8.6 \%)$. Becke98/AE and FSLYP/ECP are the methods that give the largest disagreement with the experiment: Becke98/AE overestimates $D_{e}$ by $0.221 \mathrm{eV}(11.9 \%)$, and FSLYP/ECP underestimates $D_{e}$ by $0.525 \mathrm{eV}(28.4 \%)$.

The effects of ECP and XC functionals on the dissociation energy of $\mathrm{Ni}_{2}$ do not seem to show similar trends to the ones seen for the bond length. However, similar trends can be noticed if, instead of $D_{e}$, one compares the asymptotic dissociation energy $D_{e}^{a}$, which is the dissociation energy with respect to the ${ }^{3} D$ atoms that correlate with the ground state of the nickel dimer $\left(D_{e}^{a}=D_{e}+2 E_{\mathrm{Ni}^{3} D}\right.$, where $E_{\mathrm{Ni}^{3} D}$ is the energy of the ${ }^{3} D$ state of $\mathrm{Ni}$ atom relative to the energy of the ground state).

The agreement of computed $D_{e}^{a}$ with the experimental value is clearly better than that of $D_{e}$. B3LYP/AE with $\Delta D_{e}^{a}=-0.015 \mathrm{eV} \quad(-0.8 \%)$ and B3LYP/ECP $\Delta D_{e}^{a}$ $=0.031 \mathrm{eV}(1.7 \%)$ give the best agreement with the experiment, similar to CASPT2, for which $\Delta D_{e}^{a}=0.040 \mathrm{eV}$ (2.2\%), and FSLYP/AE $\Delta D_{e}^{a}=0.052 \mathrm{eV}(2.8 \%)$. FSLYP/
ECP with $\Delta D_{e}^{a}=0.106 \mathrm{eV}(5.7 \%)$ is a little worse than FSLYP/AE. Becke98 gives the largest overestimation for $D_{e}^{a}$ : Becke98/AE gives $\Delta D_{e}^{a}=0.221 \mathrm{eV}(11.9 \%)$ and Becke98/ECP gives $\Delta D_{e}^{a}=0.349 \mathrm{eV}(18.8 \%)$.

For all three functionals, the ECP basis tends to overestimate the $D_{e}^{a}$ compared to the AE basis. For B3LYP and FSLYP the effect of ECP on $D_{e}^{a}$ is the smallest among the three functionals $(\approx 0.05 \mathrm{eV})$, while for the Becke98 functional the effect of ECP on $D_{e}^{a}$ is largest $(0.13 \mathrm{eV})$, for which $\Delta D_{e}^{a}$ increases from $0.22 \mathrm{eV}$ for $\mathrm{AE}$ to $0.35 \mathrm{eV}$ for ECP. However, for Becke98/ECP $\Delta D_{e}$ is only $-0.06 \mathrm{eV}$ due to cancellation of large and positive $\Delta D_{e}^{a}$ and the large $E\left(\mathrm{Ni}^{3} D\right)$. For FSLYP this cancellation does not happen and both FSLYP/AE and FSLYP/ECP underestimate the dissociation energy by fairly large amount because of the large error in $E\left({ }^{3} \mathrm{DNi}\right)$.

\section{Vibrational frequency}

As can be noticed in Fig. 2, there seem to be a general trend for all our DFT calculations, that the error in vibrational frequency decreases as the error in bond length increases. CASSCF/IC-ACPF is close to following the same trend, but CASPT2 is clearly an outlier.

It is apparent that all calculations included in Table II and Fig. 2-both our DFT calculations and CASPT2 and CASSCF/IAACPF wavefunction methods included for comparison-overestimate the vibrational frequency of $\mathrm{Ni}_{2}$. The deviations from the experimental value of the computed harmonic vibrational frequency, $\Delta \omega_{e}=\omega_{e}^{\text {comp }}-\omega_{e}^{\text {expt }}$ range between $10.6 \mathrm{~cm}^{-1}(4.3 \%)$ and $36.8 \mathrm{~cm}^{-1}$ (14.9\%) among our DFT results.

Becke98/AE with $\Delta \omega_{e}=10.6 \mathrm{~cm}^{-1} \quad(4.3 \%)$ and B3LYP/AE $\Delta \omega_{e}=12.7 \mathrm{~cm}^{-1}(5.2 \%)$ give the best agreement with the experiment among our DFT results, slightly worse than CASSCF/IC-ACPF, for which $\Delta \omega_{e}=6.8 \mathrm{~cm}^{-1}$ (2.8\%). Becke98/ECP follows, overestimating $\omega_{e}$ by $18.9 \mathrm{~cm}^{-1}$ (7.7\%). B3LYP/ECP and FSLYP/AE perform similarly with $\Delta \omega_{e}=23.1 \mathrm{~cm}^{-1}(9.4 \%)$ and $\Delta \omega_{e}$ $=24.9 \mathrm{~cm}^{-1}(10.1 \%)$, respectively. FSLYP/ECP with $\Delta \omega_{e}$ $=36.8 \mathrm{~cm}^{-1}(14.9 \%)$ gives the worst agreement with experiment, similar to CASPT2, which overestimates $\omega_{e}$ by $34.8 \mathrm{~cm}^{-1}(14.1 \%)$.

\section{Summary of the results for $d_{\delta}^{A} d_{\delta}^{B}$-holes states of $\mathrm{Ni}_{2}$}

All calculations predict $d_{\delta}^{A} d_{\delta}^{B}$-holes states to have the lowest energy both for singlet and for triplet spin multiplicities. The bond lengths of optimized geometries, dissociation energies and vibrational frequencies for these states calculated with the described DFT methods are tabulated in Table III for comparison.

The first observation is that the ${ }^{3}\left(d_{x^{2}-y^{2}}^{A} d_{x^{2}-y^{2}}^{B}\right)$ and/or ${ }^{3}\left(d_{x y}^{A} d_{x y}^{B}\right)$ are the highest-lying states, for all calculations, and that the spin projection changes the ordering of the singlet states for all three all-electron calculations. For these calculations, the lowest energy is obtained for the un- 
TABLE III. DFT results for $\mathrm{Ni}_{2} . d_{e}$, bond length $(\AA) ; D_{e}$, dissociation energy, relative to ground state $\mathrm{Ni}$ atoms (without zero-point correction, $\mathrm{eV}) ; \omega_{e}$, vibrational frequency $\left(\mathrm{cm}^{-1}\right)$. The notation used for the states is ${ }^{M}\left(h^{A} h^{B}\right)$, where $M$ is the multiplicity, $h^{A}$ and $h^{B}$ are the holes on Ni atoms $A$ and $B$, respectively. The $S_{z}=0,\left\langle S^{2}\right\rangle=1$ mixed states are denoted by ${ }^{1,3}\left(h^{A} h^{B}\right)$.

\begin{tabular}{|c|c|c|c|c|}
\hline Method & State & $d_{e}$ & $D_{e}$ & $\omega_{e}$ \\
\hline \multirow[t]{6}{*}{ Becke98/AE } & ${ }^{3}(d$ & 2.302 & 2.054 & 257.0 \\
\hline & & 2.298 & 2.068 & 256.8 \\
\hline & ${ }^{1,3}\left(d_{x^{2}}^{A}\right.$ & 2.298 & 2.065 & 257.0 \\
\hline & ${ }^{3}\left(d_{x^{2}-}^{A^{x^{2}}}\right.$ & 2.297 & 2.062 & 257.1 \\
\hline & & 2.296 & 2.071 & 256.8 \\
\hline & $\left.{ }_{2} d_{x^{2}-y^{2}}^{B}\right)$ & 2.299 & 2.062 & 256.9 \\
\hline \multirow[t]{9}{*}{ Becke98/ECP } & ${ }^{3}(d)$ & 2.283 & 1.779 & 266.6 \\
\hline & & 2.282 & 1.783 & 266.6 \\
\hline & & 2.280 & 1.788 & 265.3 \\
\hline & $\left.d_{x y}^{B}\right)$ & 2.279 & 1.787 & 265.9 \\
\hline & & 2.279 & 1.787 & 266.4 \\
\hline & & 2.278 & 1.787 & 265.0 \\
\hline & ${ }^{1}(d$ & 2.278 & 1.792 & 265.1 \\
\hline & $1,3\left(d_{x y}^{A}\right.$ & 2.280 & 1.783 & 265.8 \\
\hline & $1,3\left(d_{x^{2}-y^{2}}^{A} d_{x^{2}-y^{2}}^{B}\right)$ & 2.280 & 1.787 & 265.9 \\
\hline \multirow[t]{6}{*}{ B3LYP/AE } & ${ }^{3}\left(d_{x}^{A}\right.$ & 2.296 & 1.817 & 260.1 \\
\hline & & 2.293 & 1.832 & 259.2 \\
\hline & ${ }_{1,3}(c$ & 2.292 & 1.828 & 259.6 \\
\hline & ${ }^{3}(d)$ & 2.292 & 1.825 & 260.0 \\
\hline & ${ }^{1}(c$ & 2.291 & 1.835 & 258.9 \\
\hline & ${ }^{1,3}\left(d_{x^{2}-y^{2}}^{A} d_{x^{2}-y^{2}}^{B}\right)$ & 2.294 & 1.826 & 259.5 \\
\hline \multirow[t]{6}{*}{ B3LYP/ECP } & & 2.275 & 1.844 & 269.4 \\
\hline & & 2.273 & 1.848 & 267.7 \\
\hline & ${ }^{1,3}\left(d_{x^{2}}^{A}\right.$ & 2.272 & 1.850 & 268.5 \\
\hline & ${ }^{3}(c$ & 2.271 & 1.851 & 269.3 \\
\hline & $\left.2-y^{2}\right)$ & 2.271 & 1.850 & 267.6 \\
\hline & ${ }^{1,3}\left(d_{x^{2}-y^{2}}^{A} d_{x^{2}-y^{2}}^{B}\right)$ & 2.273 & 1.847 & 268.5 \\
\hline \multirow[t]{6}{*}{ FSLYP/AE } & $\left.-y^{2}\right)$ & 2.264 & 1.645 & 272.4 \\
\hline & & 2.262 & 1.662 & 271.3 \\
\hline & $\left.d_{x y}^{B}\right)$ & 2.261 & 1.656 & 271.7 \\
\hline & ${ }^{3}(d)$ & 2.261 & 1.650 & 272.2 \\
\hline & & 2.260 & 1.664 & 271.0 \\
\hline & ${ }^{1,3}\left(d_{x^{2}-y^{2}}^{A} d_{x^{2}-y^{2}}^{B}\right)$ & 2.262 & 1.654 & 271.7 \\
\hline \multirow[t]{9}{*}{ FSLYP/ECP } & ${ }^{3}\left(d_{x}^{t}\right.$ & 2.240 & 1.307 & 284.4 \\
\hline & & 2.240 & 1.299 & 284.4 \\
\hline & $\left(d_{x y}^{B}\right)$ & 2.238 & 1.319 & 283.3 \\
\hline & $1,3\left(d_{x^{2}-y^{2}}^{A} d_{x y}^{B}\right)$ & 2.237 & 1.314 & 283.8 \\
\hline & ${ }^{3}\left(d_{x^{2}-y^{2}}^{A} d_{x y}^{B}\right)$ & 2.237 & 1.309 & 284.2 \\
\hline & ${ }^{1}\left(d_{x y}^{A} d_{x y}^{B}\right)$ & 2.236 & 1.325 & 283.0 \\
\hline & ${ }^{1}\left(d_{x^{2}-y^{2}}^{A} d_{x^{2}-y^{2}}^{B}\right)$ & 2.236 & 1.318 & 283.0 \\
\hline & ${ }^{1,3}\left(d_{x y}^{A} d_{x y}^{B}\right)^{x^{2}-y^{2}}$ & 2.238 & 1.316 & 283.7 \\
\hline & $1,3\left(d_{x^{2}-y^{2}}^{A} d_{x^{2}-y^{2}}^{B}\right)$ & 2.238 & 1.308 & 283.7 \\
\hline
\end{tabular}

projected singlet ${ }^{1,3}\left(d_{x^{2}-y^{2}}^{A} d_{x y}^{B}\right)$ state, and upon projection, the degenerate ${ }^{1}\left(d_{x^{2}-y^{2}}^{A} d_{x^{2}-y^{2}}^{B}\right)$ and ${ }^{1}\left(d_{x y}^{A} d_{x y}^{B}\right)$ become the ground state.

For the B3LYP/ECP calculation spin projection does not change the ${ }^{3}\left(d_{x^{2}-y^{2}}^{A} d_{x y}^{B}\right)$ ground state, although it makes the ${ }^{3}\left(d_{x^{2}-y^{2}}^{A} d_{x y}^{B}\right)$ ground state nearly degenerate with the degenerate ${ }^{1}\left(d_{x^{2}-y^{2}}^{A} d_{x^{2}-y^{2}}^{B}\right)$ and ${ }^{1}\left(d_{x y}^{A} d_{x y}^{B}\right)$. However, the ${ }^{3}\left(d_{x^{2}-y^{2}}^{A} d_{x y}^{B}\right)$ ground state is only $0.001 \mathrm{eV}$ lower in energy than the degenerate ${ }^{1}\left(d_{x^{2}-y^{2}}^{A} d_{x^{2}-y^{2}}^{B}\right)$ and ${ }^{1}\left(d_{x y}^{A} d_{x y}^{B}\right)$. For Becke98/ECP the unprojected ground state is ${ }^{1,3}\left(d_{x^{2}-y^{2}}^{A} d_{x y}^{B}\right)$ degenerate with ${ }^{3}\left(d_{x^{2}-y^{2}}^{A} d_{x y}^{B}\right)$, and upon spin projection, ${ }^{1}\left(d_{x^{2}-y^{2}}^{A} d_{x^{2}-y^{2}}^{B}\right)$ becomes the ground state, with a dissociation energy larger than the one of ${ }^{1}\left(d_{x y}^{A} d_{x y}^{B}\right)$ by $0.005 \mathrm{eV}$. For FSLYP/ECP the ${ }^{1,3}\left(d_{x y}^{A} d_{x y}^{B}\right)$ unprojected ground state does not change upon spin projection, but the difference between the $D_{e}$ of ${ }^{1}\left(d_{x^{2}-y^{2}}^{A} d_{x^{2}-y^{2}}^{B}\right)$ and that of ${ }^{1}\left(d_{x y}^{A} d_{x y}^{B}\right)$ is the largest among all calculations: $0.008 \mathrm{eV}$, and is larger than the numerical accuracy of the DFT calculations (better than 0.1 $\mathrm{meV})$.

It is also worth noting that for all calculations the average $D_{e}$ of singlet states is larger than the one of the triplet states. However, the difference between the singlet and the triplet is very small for Becke98/ECP and B3LYP/ECP $(0.006 \mathrm{eV}$ and $0.003 \mathrm{eV}$, respectively). For the other calculations, the difference is somewhat larger, around $0.015 \mathrm{eV}$.

However, it is important to note from Table III that for each combination of exchange-correlation functional and basis set used, all $\delta \delta$-holes states are in a very narrow energy range: $\approx 20 \mathrm{meV}$ for all all-electron calculations, $26 \mathrm{meV}$ for FSLYP/ECP, $13 \mathrm{meV}$ for Becke98/ECP and only $7 \mathrm{meV}$ for B3LYP/ECP.

Since, as shown above, the ordering of states can change upon spin projection, if possible to perform, spin projection is desirable. However, we want to emphasize that the differences between the lowest broken-symmetry singlet states and the projected singlet ground states, for the all-electron calculations and FSLYP/ECP, is less than $10 \mathrm{meV}$, and for some applications that difference may not be relevant. Nevertheless, we plan to consider spin projection for larger clusters, if possible, at least for evaluating the errors that arise from it.

\section{Potential energy curves (PEC)}

In order to determine the ground state of $\mathrm{Ni}_{2}$ we did a full scan of the PEC for each method and for each unique combination of holes. All calculations predict $\delta \delta$-holes states to have the lowest energy, with the next level $50-100 \mathrm{meV}$ above, $\sigma \delta$ for Becke98, and B3LYP calculations and $\pi \delta$ for FSLYP calculations.

The computations of $\sigma \pi$ states with Becke98 and B3LYP functionals only converge to $10^{-5}-10^{-4}$ hartree within 100 iterations in the 1.95-2.55 $\AA$ range. Because the FSLYP calculations, which converge properly, predict that these states are $\approx 200 \mathrm{meV}$ higher, the same value as the "not-so-converged" results for the above calculations, we have chosen not to investigate the matter any further.

Since the results of the PEC scans are rather similar, and B3LYP is our functional of choice, in the following discussion of the PEC's, we focus attention principally on the results from B3LYP calculations.

The B3LYP/AE and B3LYP/ECP PEC of singlet $\left(S_{z}\right.$ $=0$ ) and triplet $\left(S_{z}=1\right)$ states of $\mathrm{Ni}_{2}$ (both unrestricted, symmetry broken) are shown in Figs. 3 and 4, respectively, along with the variation of $\left\langle S^{2}\right\rangle$ with the bond length for all possible positions of holes in the $3 d$ shell on both atoms, grouped by hole type. The first trend that can be noticed is that the equilibrium bond length increases as the dissociation 

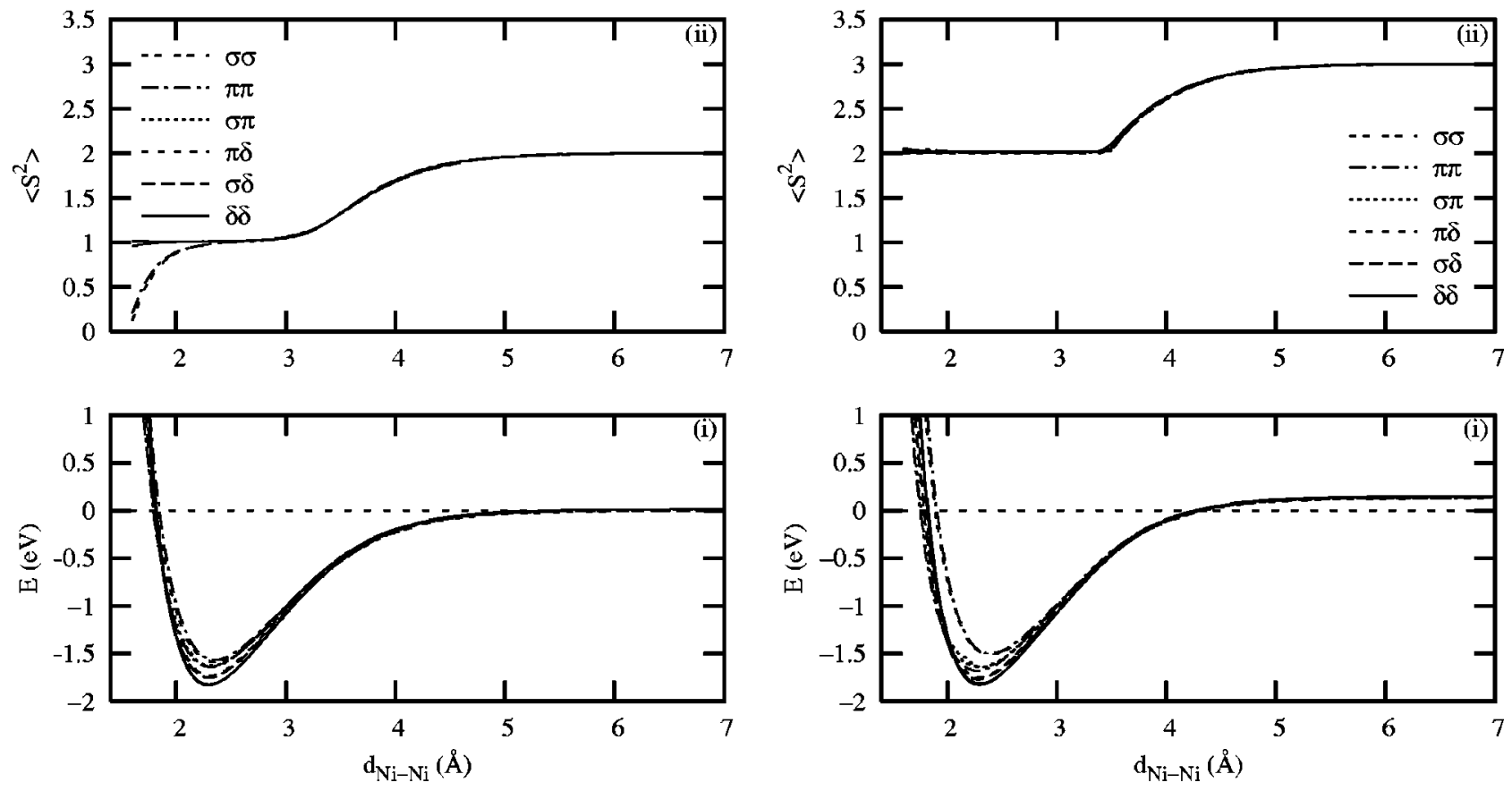

(a)

$$
\text { singlet }
$$

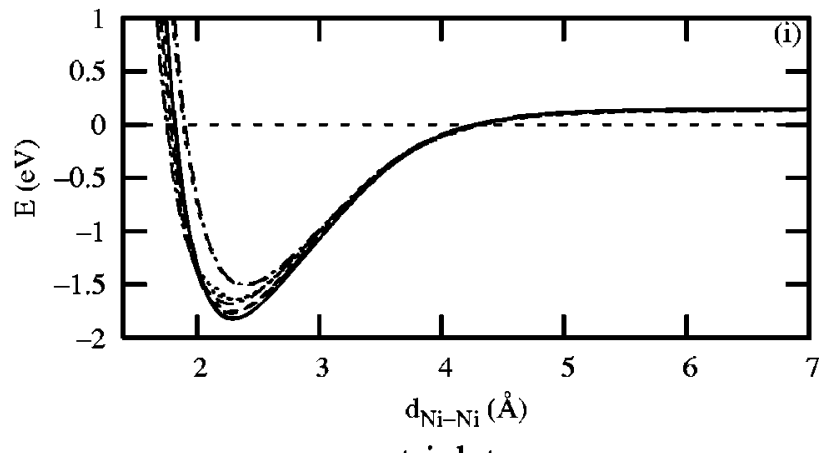

(b)

triplet

FIG. 3. B3LYP/Wachters $+f$ PECs of $\mathrm{Ni}_{2}$. Energy in $\mathrm{eV}$, relative to ground state $\mathrm{Ni}$ atoms and bond length in $\AA$.

energy decreases. Aside for a few states (singlet $\sigma \sigma$ and $\pi \pi$ ), all states have $\left\langle S^{2}\right\rangle \approx 1$ over a large interval, validating the weakly interacting $3 d^{9}$ units model for a large range of bond lengths. Even the singlet $\sigma \sigma$ and $\pi \pi$ states have $\left\langle S^{2}\right\rangle \approx 1$ in a range of about $\pm 1 \AA$ around the equilibrium bond length.

One can notice a big difference between AE and ECP PEC's: ECP PEC's branch around $3.5 \AA$. There are two causes for branching: one, which is not related to functional $^{74}$ or ECP, is the restricted-unrestricted crossover, while the other cause is dissociation into ${ }^{3} F$ ground state of $\mathrm{Ni}$ atoms. These two effects overlap because the branching is obtained by scanning the PEC from $\approx 3.5 \AA$, increasing the bond length and using as initial guess the molecular orbitals from the previous calculation. Depending on the initial guess, the calculation may end in the restricted or unrestricted solution, or, at large distances, the calculation may
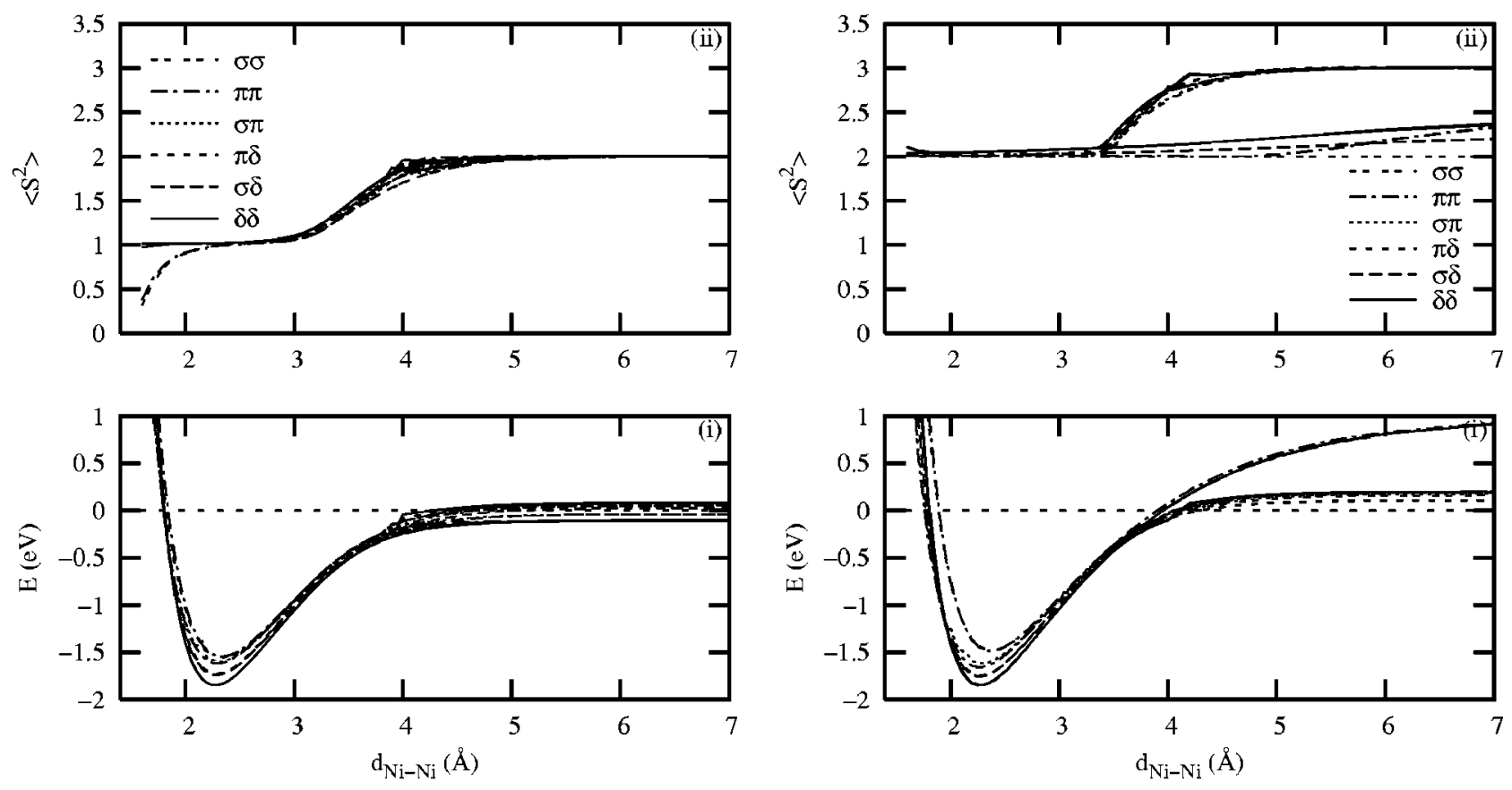

(a)

singlet

(b) triplet

FIG. 4. B3LYP/Stuttgart RSC ECP PECs of $\mathrm{Ni}_{2}$. Energy in eV, relative to ground state $\mathrm{Ni}$ atoms and bond length in $\AA$. 
TABLE IV. Ground state of NiH-comparison between computations and experiment. $d_{e}$, bond length $(\AA)$; $D_{e}$, dissociation energy, relative to ground state $\mathrm{Ni}$ atoms (without zero-point correction, $\mathrm{eV}$ ); $\omega_{e}$, vibrational frequency $\left(\mathrm{cm}^{-1}\right)$; and $\mu$, dipole moment (Debye). The relative errors with respect to experimental values are given in parentheses, and the average (AARD) and the maximum (MARD) absolute relative deviations from experimental values of $d_{e}, D_{e}, \omega_{e}$, and $\mu$ are listed under the AARD and MARD columns, respectively.

\begin{tabular}{|c|c|c|c|c|c|c|}
\hline Method & $d_{e}$ & $D_{e}$ & $\omega_{e}$ & $\mu$ & AARD & MARD \\
\hline B3LYP/ECP & $1.454(-1.6)$ & 2.901 (13.8) & $1937.6(-0.2)$ & $2.29(-12.7)$ & 7.1 & 13.8 \\
\hline Becke98/ECP & $1.456(-1.4)$ & $2.808(10.1)$ & $1927.6(-0.7)$ & $2.43(-7.2)$ & 4.8 & 10.1 \\
\hline B3LYP/AE & $1.474(-0.2)$ & $2.856(12.0)$ & $1940.2(-0.1)$ & $2.43(-7.1)$ & 4.8 & 12.0 \\
\hline FSLYP/AE & $1.470(-0.5)$ & $2.681(5.1)$ & $1943.8(0.1)$ & $2.91(11.2)$ & 4.2 & 11.2 \\
\hline CASPT $^{\mathrm{a}}$ & $1.463(-0.9)$ & $2.76(8.2)$ & $2022.3(4.2)$ & $2.54(-3.1)$ & 4.1 & 8.2 \\
\hline Becke98/AE & $1.477(0.0)$ & $2.888(13.3)$ & $1944.2(0.1)$ & $2.59(-1.0)$ & 3.6 & 13.3 \\
\hline FSLYP/ECP & $1.449(-1.9)$ & $2.526(-0.9)$ & $1953.4(0.6)$ & $2.74(4.5)$ & 2.0 & 4.5 \\
\hline Expt. $^{\mathrm{b}}$ & 1.477 & 2.55 & 1941.3 & $2.6(3.8)$ & & \\
\hline
\end{tabular}

${ }^{a}$ We report here the values from Table VI of Ref. 67 for $d_{e}, D_{e}$, and $\omega_{e}$, and from Table VII for $\mu$ $[\mathrm{PT} 2 \mathrm{~F}(3 s 3 p)+\mathrm{RC}]$, from which we subtract the estimated RC. From the same reference, we estimate the RC to $d_{e}$ and $D_{e}$ from Table $\mathrm{V}$ and the RC to $\mu$ from Table VII, as the difference between the PT2F+ RC values and the PT2F ones. We use the MRCI RC to $\omega_{e}$ from Ref. 78. We also subtract these relativistic corrections from the experimental values.

${ }^{\mathrm{b}}$ Experimental values from which we subtract the CASPT2 RC to $d_{e}, D_{e}$, and $\mu$ from Ref. 67, and the MRCI RC to $\omega_{e}$ from Ref. 78 (see footnote a). The experimental value of $d_{e}$ is $1.454 \AA$ [75, cited in Ref. 67], from which we subtract the CASPT2 RC of $-0.023 \AA$; the experimental value of $D_{e}$ is $2.70 \mathrm{eV}$ [recommended value from Ref. 76, cited in Ref. 67], from which we subtract the CASPT2 RC of $0.15 \mathrm{eV}$; the experimental value of $\omega_{e}$ is $2001.3 \mathrm{~cm}^{-1}$ [75, cited in Ref. 67], from which we subtract the MRCI RC of $60 \mathrm{~cm}^{-1}$; the experimental value of $\mu$ is $2.4 \pm 0.1$ Debye [77, cited in Ref. 67], from which we subtract the CASPT2 RC of -0.22 Debye.

converge to the ${ }^{3} F+{ }^{3} F,{ }^{3} F+{ }^{3} D$ or ${ }^{3} D+{ }^{3} D$ states of the Ni atoms. The restricted-unrestricted branching is likely to show up for any of the methods, but the ground state branching can only appear for the methods that predict ${ }^{3} F$ ground state for $\mathrm{Ni}$, namely FSLYP/AE FSLYP/ECP, and Becke98/ECP along with the discussed B3LYP/ECP.

One can also notice that some of the B3LYP/ECP PEC's have asymptotes below 0 , i.e., below the energy of the ground state of the two nickel atoms. A closer look reveals that the asymptotes of the $d_{x y} d_{x z^{-}}, d_{x y} d_{y z^{-}}, d_{x z} d_{y z^{-}}, d_{x z} d_{x z^{-}}$, $d_{y z} d_{y z^{-}}, d_{x y} d_{x y^{-}}, d_{x^{2}-y^{2}} d_{x z^{-}}, d_{x^{2}-y^{2}} d_{y z^{-}}, d_{x^{2}-y^{2}} d_{x y^{-}}$, and $d_{x^{2}-y^{2}} d_{x^{2}-y^{2}}$-holes states lie $0.1 \mathrm{eV}$ below the ground state of the two nickel atoms, the ones of $d_{z^{2}} d_{x z^{-}}, d_{z^{2}} d_{y z^{-}}, d_{z^{2}} d_{x y^{-}}$, and $d_{z^{2}} d_{x^{2}-y^{2}}$-holes states lie $0.04 \mathrm{eV}$ below the ground state of the two nickel atoms, and only $d_{z^{2}} d_{z^{2}}$-holes state lies 0.03 $\mathrm{eV}$ above the ground state of the two nickel atoms. The most likely explanation for this observation is that B3LYP/ECP predicts a lower energy for a state that is not in the space of states spanned by our initial guess. This issue needs further investigation, but since the effect is rather small (at most $0.05 \mathrm{eV} /$ nickel atom), we chose to investigate the issue in a further paper.

The initial PEC scans are done either with broken symmetry atomic initial guess $\left(3 d^{9} 4 s^{1} \uparrow \uparrow+\downarrow \downarrow 3 d^{9} 4 s^{1}\right.$ for singlet and $3 d^{9} 4 s^{1} \uparrow \uparrow+\downarrow \uparrow 3 d^{9} 4 s^{1}$ for triplet) at each bond length or, starting from $10 \AA$ and decreasing the bond length and using as initial guess the molecular orbitals at the previous bond length. Either initial guess gives the same results, but the method using atomic initial guess needs a few extra iterations. For larger cluster calculations it may be useful to save the molecular orbitals at each geometry configuration and try to reuse them for a neighboring point calculation.

It is apparent from Fig. 3 that for the B3LYP/AE calculation the singlet dissociates to the correct $2^{3} D$ atoms limit $\left(\left\langle S^{2}\right\rangle=2\right)$, whereas the triplet dissociates to ${ }^{3} D+{ }^{1,3} D$, which is $0.14 \mathrm{eV}$ above the correct limit. This type of error only plays an important role at large distances, when the molecule starts to resemble two separated atoms, and can be correlated with $\left\langle S^{2}\right\rangle$ of the Kohn-Sham determinant. When $\left\langle S^{2}\right\rangle$ is close to the exact value, this type of error is not present. For $\mathrm{Ni}_{2}$, both singlet and triplet, the $\left\langle S^{2}\right\rangle$ is correct (i.e. equal to the theoretical value) for $2 \AA<d_{e}<3 \AA$. At interatomic distances greater than $\approx 3 \AA,\left\langle S^{2}\right\rangle$ starts to increase, and so does the error in the energy of the triplet. At interatomic distances larger than approximately $\left.\approx 4 \AA . \AA S^{2}\right\rangle$ for the triplet reaches a value of $\approx 3$ and stays constant for larger distances. Similarly, the error in the energy of the triplet approaches the asymptotic value of $0.14 \mathrm{eV}$.

In larger clusters, this could be a potential issue for computing barriers. However, only configurations in which one atom is at sufficiently large distance from other atoms, completely or partly detached (evaporated) from the cluster, and in the ${ }^{1,3} D$ state, would encounter the above described problem. Moreover, the error $(\leqslant 0.14 \mathrm{eV})$ could be important if the height of the barrier were small. But the evaporation energy of an atom from the cluster is likely to be of the same order of magnitude as the dissociation energy of the dimer $(\approx 1.5 \mathrm{eV})$, and the height of the barrier would be overestimated by $\approx 10 \%$. Consequently, this error should be unimportant for large clusters.

\section{Nickel hydride}

The bond lengths $d_{e}$, dissociation energies $D_{e}$, vibrational frequencies $\omega_{e}$, and dipole moment $(\mu)$ for ground states of $\mathrm{NiH}$ from different calculations are reported in Table IV along with experimental values and results from 


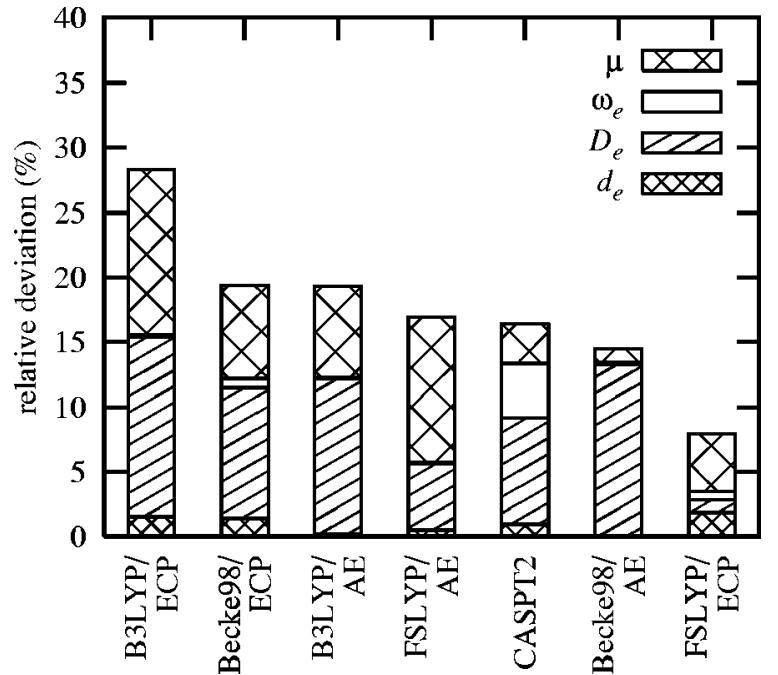

FIG. 5. The absolute relative deviations from experiment of computed dissociation energy $D_{e}$, bond length $d_{e}$, vibrational frequency $\omega_{e}$, and dipole moment $\mu$ for $\mathrm{NiH}$. The results are arranged from left to right in order of decreasing TARD - the sum of absolute relative deviations from the experimental values of the computed $d_{e}, D_{e}, \omega_{e}$, and $\mu$.

other theoretical studies, listed in the order of decreasing AARD from the experimental values of the computed $d_{e}$, $D_{e}, \omega_{e}$, and $\mu$.

The experimental values reported in Table IV are the deperturbed values of $d_{e}$ and $\omega_{e}$ of Gray et al. [Ref. 75 cited in Ref. 67], the recommended value of $D_{e}$ from Ref. 76 (cited in Ref. 67) and $\mu$ from Ref. 77 (cited in Ref. 67), from which we subtract the CASPT2 RC to $d_{e}, D_{e}$, and $\mu$ from Ref. 67, and the MRCI RC to $\omega_{e}$ from Ref. 78 (see footnote a of Table IV for details).

The absolute relative deviations from the experimental values of the computed bond lengths $d_{e}$, dissociation energies $D_{e}$, vibrational frequencies $\omega_{e}$, and dipole moment $\mu$ of the ground state of $\mathrm{NiH}$ are plotted in Fig. 5 for comparison. They are arranged from left to right in order of decreasing TARD - the sum of absolute relative deviations from the experimental values of the computed $d_{e}, D_{e}, \omega_{e}$, and $\mu$.

From Fig. 5, as well as from Table IV, it is apparent that for $\mathrm{NiH}$, the best overall agreement with experiment among our DFT calculations is obtained for FSLYP/ECP (7.9\% TARD), followed by Becke98/AE (14.4\% TARD) and FSLYP/AE (16.9\% TARD) similar to CASPT2 (16.4\% TARD). B3LYP/AE (19.3\% TARD) is next, similar to Becke98/ECP (19.4\% TARD), and B3LYP/ECP (28.3\% TARD) gives the largest disagreement with experiment.

All our DFT calculations predict ${ }^{2} \Delta$ ( $\delta$-hole $)$ ground state, in agreement with the CASPT2 calculation and experiment. However, it is important to note, that, for Becke98/ ECP and FSLYP/ECP results the difference between the $d_{x y}$-hole and the $d_{x^{2}-y^{2}}$-hole components of the ${ }^{2} \Delta$ state-2 $\mathrm{meV}$ and $5 \mathrm{meV}$, respectively-is larger than the error of the DFT calculations $(\leqslant 0.1 \mathrm{meV})$. We report the energy of the component with the lowest energy as the energy of the ground state.

It is apparent that all calculations included in Table IV and Fig. 6 underestimate the bond length of $\mathrm{NiH}$.

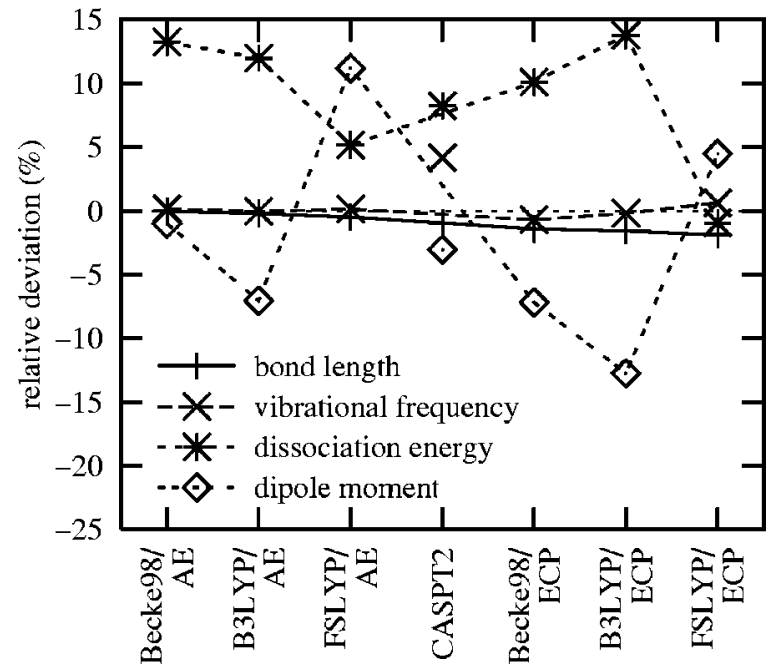

FIG. 6. The relative deviations of computed bond length, dissociation energy, vibrational frequency and dipole moment from experimental values for $\mathrm{NiH}$. Only the results from our DFT calculations are connected by lines. CASPT2 values are included for comparison.

Becke98/AE with $\quad \Delta d_{e}=d_{e}^{\text {comp }}-d_{e}^{\text {expt }}=-0.0003 \AA$ $(-0.02 \%)$ gives the best agreement with experiment. The other DFT calculations and CASPT2 give significantly shorter bond lengths for $\mathrm{NiH}$ than Becke98/AE, but they can still be considered in good agreement with the experiment, giving $\Delta d_{e}$ ranging from $-0.003 \AA(-0.2 \%)$ for B3LYP/AE to $-0.028 \AA(-1.9 \%)$ for FSLYP/ECP. Among all three $\mathrm{XC}$ functionals, the best agreement with experiment for the bond length is obtained with the Becke98 functional, both AE and ECP. B3LYP follows with a bond length 0.003 $\AA$ shorter than the one computed with Becke98. FSLYP bond length is in the worst agreement with the experiment, but only $\approx 0.004 \AA$ longer than the B3LYP bond length. For each of the three XC functionals used, ECP calculation predicts shorter bond length than the $\mathrm{AE}$ one by $\approx 0.02 \AA$, like in the case of $\mathrm{Ni}_{2}$, but this worsens the agreement with the experiment, unlike in the case of $\mathrm{Ni}_{2}$.

The computed dissociation energies span a large range of values, from $2.53 \mathrm{eV}$ for FSLYP/ECP to $2.90 \mathrm{eV}$ for B3LYP/ ECP. Among all DFT computations, only FSLYP/ECP underestimates $D_{e}$ by $0.024 \mathrm{eV}(0.9 \%)$, and gives the best agreement with the experiment. All other DFT computations and CASPT 2 overestimate $D_{e}$ : FSLYP/AE by $5 \%$, CASPT 2 by $8 \%$, and Becke98/ECP, B3LYP/AE, Becke98/AE, and B3LYP/ECP by $10 \%, 12 \%, 13 \%$, and $14 \%$, respectively, giving the largest disagreement with experiment. Like in the case of $\mathrm{Ni}_{2}$, the effects of ECP and XC functionals on the dissociation energy of $\mathrm{Ni}_{2}$ do not seem to show similar trends to the ones seen for the bond length. It can be verified that trends show up upon correcting $D_{e}$ with the energy of the ${ }^{3} \mathrm{D}$ state of $\mathrm{Ni}$, but, since for $\mathrm{NiH}$ there is no physical ground for that kind of correction, we chose not to do it. However, it is worth noting that the errors in the atomic energies have such large influence on the energetics of molecules.

The differences in the theoretical harmonic vibrational frequencies compared to the experimental values are less 
than $1 \%$ for our DFT calculations, while CASPT2 has the largest difference from the experimental value among the results plotted in Fig. 6 and listed in Table IV.

For $\mathrm{NiH}$ the dipole moment can be expected to be a more sensitive measure of the quality of the method, ${ }^{67}$ and a comparison of the theoretical and experimental values of the dipole moment listed in Table IV and plotted in Fig. 6 shows that Becke98/AE gives the best agreement, similar to CASPT2. B3LYP/AE underestimates the dipole moment by $7 \%$ and FSLYP/AE overestimates it by a large amount $(11 \%)$. ECP have a strong effect on $\mu$, lowering its value by $\approx 0.15 D(6 \%)$, bringing FSLYP/ECP in closer agreement with experiment and worsening the agreement for B3LYP and Becke98. It is worth noting that Becke98 predicts a value for $\mu$ in better agreement with the experiment than B3LYP. Since $\mu$ is a one-electron property, this may be an indication that Becke98 gives a more accurate ground state electron density.

\section{CONCLUSION}

We have used DFT with hybrid exchange-correlation functionals in the broken-symmetry unrestricted formalism to study the electronic structure of nickel dimer and nickel hydride as model systems for larger bare/hydrogenated nickel clusters. We have examined three hybrid functionals: the popular B3LYP, Becke's newest optimized functional Becke98, and the simple FSLYP functional (50\% HartreeFock and 50\% Slater exchange and LYP gradient-corrected correlation functional) with two basis sets: all-electron (AE) Wachters $+f$ basis set and Stuttgart RSC ECP and basis set.

For $\mathrm{Ni}_{2}$, all of our DFT calculations give bond lengths that are within $0.1 \AA$ (5\%) from the experimental value, and in good agreement with the high-level wavefunction methods CASPT $^{67}$ and CASSCF/IC-ACPF. ${ }^{72}$ Only Becke98/AE and $\mathrm{B} 3 \mathrm{LYP} / \mathrm{AE}$ give harmonic vibrational frequencies that are within 5\% from the experimental value, similar to CASSCF/ IC-ACPF. Becke98/ECP, B3LYP/ECP and FSLYP/AE give $\omega_{e}$ within $10 \%$ from the experimental value, similar to CASPT2, and FSLYP/ECP overestimates the experimental $\omega_{e}$ by $15 \%$. The discrepancies between calculated and experimental values of dissociation energy span a large range, between $-28 \%$ and $12 \%$. B3LYP/ECP, B3LYP/AE, and Becke98/ECP give values of $D_{e}$ that are within less than 5\% from the experimental value, similar to CASPT2. FSLYP/ $\mathrm{AE}$, and Becke98/AE give values of $D_{e}$ that are a within $12 \%$ from experimental value, similar to CASSCF/IC-ACPF. FSLYP/ECP gives a value of $D_{e}$ that is smaller than the experimental value by $28 \%$.

For $\mathrm{NiH}$, all of our DFT calculations give bond lengths that are within $0.03 \AA$ ( $2 \%$ ) from the experimental value, and in good agreement with CASPT2. ${ }^{67}$ They also give harmonic vibrational frequencies that are within less than $15 \mathrm{~cm}^{-1}$ (1\%) from the experimental value, in better agreement with experiment than CASPT2, which overestimates $\omega_{e}$ by $4 \%$. The discrepancies between the calculated and the experimental values of dissociation energy span a large range for $\mathrm{NiH}$ like they do for $\mathrm{Ni}_{2}$. FSLYP/ECP underestimates $D_{e}$ by $1 \%$, giving the best agreement with the experiment. All other DFT calculations and CASPT2 overestimate $D_{e}$ by amounts between $5 \%$ and $15 \%$. For the dipole moment the deviations from the experimental value span the largest range: between $-13 \%$ for B3LYP/ECP and 11\% for FSLYP/AE. Underestimating it by $1 \%$, Becke98/AE gives the best agreement with the experiment for the dipole moment of $\mathrm{NiH}$, similar to CASPT2, which underestimates it by $3 \%$.

We also find that for $\mathrm{Ni}_{2}$, the spin projection for the broken-symmetry unrestricted singlet states changes the ordering of the states, but the splittings are less than $10 \mathrm{meV}$. All our calculations predict a $\delta \delta$-hole ground state for $\mathrm{Ni}_{2}$ and $\delta$-hole ground state for $\mathrm{NiH}$. Upon spin projection of the singlet state of $\mathrm{Ni}_{2}$, almost all of our calculations: Becke98 and FSLYP both AE and ECP and B3LYP/AE predict ${ }^{1}\left(d_{x^{2}-y^{2}}^{A} d_{x^{2}-y^{2}}^{B}\right)$ or ${ }^{1}\left(d_{x y}^{A} d_{x y}^{B}\right)$ ground state, which is a mixture of ${ }^{1} \Sigma_{g}^{+}$and ${ }^{1} \Gamma_{g}$. B3LYP/ECP predicts a ${ }^{3}\left(d_{x^{2}-y^{2}}^{A} d_{x y}^{B}\right)$ (mixture of ${ }^{3} \Sigma_{g}^{-}$and ${ }^{3} \Gamma_{u}$ ) ground state virtually degenerate with the ${ }^{1}\left(d_{x^{2}-y^{2}}^{A} d_{x^{2}-y^{2}}^{B}\right) /{ }^{1}\left(d_{x y}^{A} d_{x y}^{B}\right)$ state, which is only 1 meV higher in energy than ${ }^{3}\left(d_{x^{2}-y^{2}}^{A} d_{x y}^{B}\right)$ ground state. The doublet $\delta$-hole ground state of $\mathrm{NiH}$ predicted by all our calculations is in agreement with the experimentally predicted ${ }^{2} \Delta$ ground state. For $\mathrm{Ni}_{2}$, all our results are consistent with the experimentally predicted ground state of $0_{g}^{+}$(a mixture of ${ }^{1} \Sigma_{g}^{+}$and ${ }^{3} \Sigma_{g}^{-}$) or $0_{u}^{-}$(a mixture of ${ }^{1} \Sigma_{u}^{-}$and ${ }^{3} \Sigma_{u}^{+}$).

The goal of this paper is to establish what might comprise a minimally reliable method for more extensive nickel cluster calculations. Since none of the studied methods gives a good agreement with experiment for all computed molecular properties of $\mathrm{Ni}_{2}$ and $\mathrm{NiH}$, we devise an ad hoc quality indicator that we name overall discrepancy $Q$, and we calculate it with the formula:

$$
Q=\underbrace{\frac{1}{7} \sum_{i}\left|\epsilon_{i}\right|}_{Q_{A}}+\underbrace{\frac{1}{21} \sum_{i<j}\left|\epsilon_{i}-\epsilon_{j}\right|}_{Q_{D}},
$$

where $i$ runs over all seven computed molecular properties for $\mathrm{Ni}_{2}$ and $\mathrm{NiH}\left(d_{e}, D_{e}\right.$, and $\omega_{e}$ of both $\mathrm{Ni}_{2}$ and $\mathrm{NiH}$, and $\mu$ of $\mathrm{NiH}) ; \epsilon_{i}$ is the relative deviation from the experimental value of the molecular property $i ; i<j$ stands for $i, j$ running over all 21 unique pairs.

The overall discrepancy $Q$ is the sum of two contributions: the average discrepancy $Q_{A}$, which measures the overall (average) deviation of the computed molecular properties from the experimental values, and the consistency $Q_{D}$, which measures the consistency of the methods both when computing different molecular properties of the same molecule (e.g., $d_{e}$ and $\omega_{e}$ of $\mathrm{Ni}_{2}$ ), and when computing molecular properties for different molecules (e.g., $d_{e}$ of $\mathrm{Ni}_{2}$ and $d_{e}$ of $\mathrm{NiH})$. For analysis, we calculate each of the indicators $Q$, $Q_{A}$, and $Q_{D}$ for each of the molecules, by partitioning Eq. (4) into the components for $\mathrm{Ni}_{2}\left(Q^{\mathrm{Ni}_{2}}, Q_{A}^{\mathrm{Ni}_{2}}\right.$, and $\left.Q_{D}^{\mathrm{Ni}_{2}}\right)$, the components for $\mathrm{NiH}\left(Q^{\mathrm{NiH}}, Q_{A}^{\mathrm{NiH}}\right.$, and $\left.Q_{D}^{\mathrm{NiH}}\right)$, and the mixed components of $Q_{D}, Q_{D}^{\mathrm{Ni}_{2}-\mathrm{NiH}^{2}}=\frac{1}{21} \Sigma_{i<j}\left|\epsilon_{i}-\epsilon_{j}\right|$ with $i$ running over the molecular properties of $\mathrm{Ni}_{2}$ and $j$ running over the ones of $\mathrm{NiH}$.

In Fig. 7 we plot the overall discrepancy $Q$ along with its components, and the maximum absolute relative deviations from experimental values (MARD) for all computed molecular properties of $\mathrm{Ni}_{2}\left(\Delta_{\max }^{\mathrm{Ni}_{2}}\right)$ and $\mathrm{NiH}\left(\Delta_{\max }^{\mathrm{NiH}}\right)$. 


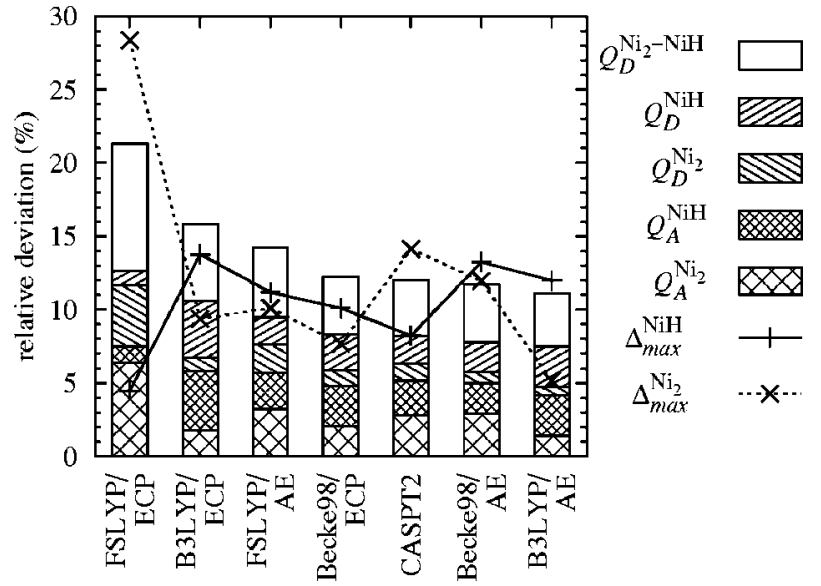

FIG. 7. Overall performance of the studied DFT methods. Total values of the overall discrepancy $Q$ are plotted, as the total heights of the bars, along with its components (see text for definition). Maximum absolute relative deviations from experimental values for all computed molecular properties of $\mathrm{Ni}_{2}\left(\Delta_{\max }^{\mathrm{Ni}_{2}}\right)$ and $\mathrm{NiH}\left(\Delta_{\text {max }}^{\mathrm{NiH}}\right)$ are also shown.

Figure 7 reveals that B3LYP/AE gives the lowest overall discrepancy $(Q=11.2 \%)$, but followed closely by Becke98/AE and Becke98/ECP with a value of $Q$ larger than the one of B3LYP/AE by only $0.5 \%$ and $1 \%$, respectively. They are also at the same overal quality as CASPT2, for which $Q=12.0 \%$. FSLYP/AE, with $Q=14.2 \%$ is a little worse than B3LYP/AE and Becke98/AE. It is apparent from Fig. 7 that the use of ECP worsen the overall agreement with experiment. The largest effect of the ECP's is on the results obtained with the FSLYP functional, increasing the value of $Q$ by $7.1 \%$. The effect is much smaller on B3LYP, increasing $Q$ by $4.6 \%$, and negligible on Becke98 (0.5\%).

It can be noticed that for most of the calculations included in Fig. 7, the value of $Q$ is close to the values of MARD for both $\mathrm{NiH}$ and $\mathrm{Ni}_{2}$. Two methods for which that is not the case are worth mentioning: B3LYP/AE and FSLYP/ECP. Both perform significantly better for one of the molecules than for the other, probably by accident. B3LYP/AE performs clearly better for $\mathrm{Ni}_{2}$ than for $\mathrm{NiH}$, but its MARD for $\mathrm{NiH}$ agrees with $Q$, while FSLYP/ECP performs much better for $\mathrm{NiH}$ than for $\mathrm{Ni}_{2}$, and its MARD for $\mathrm{Ni}_{2}$ is significantly larger than $Q$ (by 7.1\%). Thus, FSLYP/ $\mathrm{ECP}$ is the only method that is not advisable to use for bare/ hydrogenated nickel clusters. However, we want to emphasize that the methods that give the best agreement with experiment and CASPT2, B3LYP/AE, Becke98/AE, and Becke98/ECP are the methods of choice.

Our results indicate that DFT, with the B3LYP (using the Wachters $+f$ all-electron basis set) and Becke98 (using either Wachters $+f$ all-electron basis set or Stuttgart RSC effective core potential and basis set) hybrid exchange-correlation functionals in the broken-symmetry unrestricted formalism, becomes both an efficient and reliable method for predicting electronic structure of our model $\mathrm{Ni}_{2}$ and $\mathrm{NiH}$ systems, although it is far from being a black box method.

\section{ACKNOWLEDGMENTS}

The authors gratefully acknowledge support from the National Science Foundation through equipment award
TABLE V. Averages of charge density fit errors for B3LYP/AE optimizations and frequency calculations for the $12\left(d_{\delta}^{A} d_{\delta}^{B}\right)$ singlet and triplet states of $\mathrm{Ni}_{2}$ computed with and without charge density fitting. $d_{e}(\mathrm{~m} \AA)$, bond length; $E_{e}$ (mHartree), total energy; $\omega_{e}\left(\mathrm{~cm}^{-1}\right)$, vibrational frequency; $E_{e}$ $(\mathrm{meV})$, relative energies with respect to the ground state Ni atom; $\Delta E_{e}$ $(\mathrm{meV})$, relative energies with respect to the lowest energy state from each type of calculation. Mean, mean of the differences between the computations with charge density fitting and those without; Stdev, standard deviation of the differences; Max, maximum absolute difference; and RMS, the rootmean square of the differences.

\begin{tabular}{lccccc}
\hline \hline & $\begin{array}{c}d_{e} \\
\mathrm{~m}\end{array}$ & $\begin{array}{c}E_{e} \\
\text { mHartree }\end{array}$ & $\begin{array}{c}\omega_{e} \\
\mathrm{~cm}^{-1}\end{array}$ & $\begin{array}{c}E_{e} \\
\mathrm{meV}\end{array}$ & $\begin{array}{c}\Delta E_{e} \\
\mathrm{meV}\end{array}$ \\
\hline Mean & 0.30 & -0.6476 & -0.28 & -4.34 & -0.07 \\
Stdev & 0.02 & 0.0018 & 0.02 & 0.05 & 0.05 \\
Max & 0.32 & 0.6497 & 0.30 & 4.40 & 0.12 \\
RMS & 0.12 & 0.2644 & 0.12 & 1.77 & 0.03 \\
\hline \hline
\end{tabular}

(Grant No. CHE-01-31114) and from DOE Grant No. DEFG02-03ER46074. Electronic structure calculations were performed with NWCHEM Versions 4.0.1, 4.1, and 4.5 (Version 4.1 with Becke98 functional patched), as developed and distributed by Pacific Northwest National Laboratory, P.O. Box 999, Richland, WA 99352, and funded by the U.S. Department of Energy. The authors gratefully acknowledge the support of Brown University's Center for Advanced Scientific Computation and Visualization in the present research. One of the authors (C.V.D.) would also like to thank Dr. Richard L. Martin for useful suggestions, and Dr. Cristian Predescu and Dr. Dubravko Sabo for helpful discussions.

\section{APPENDIX A: ACCURACY OF CHARGE DENSITY FITTING}

As stated in Sec. II we use charge density fitting for the calculations using the all-electron Wachters $+f$ basis set, for which we employ the Ahlrichs Coulomb Fitting ${ }^{56,57}$ basis set. For evaluating the error introduced by charge density fitting we perform the atomic computations with B3LYP functional and Wachters $+f$ basis set with and without charge density fitting. The charge density fitting lowers the total energies of computed atomic states by $2.5-3 \times 10^{-4}$ hartree. The errors in the relative energies are less severe, ranging from -3.8 to $1.5 \mathrm{meV}$.

To be cautious, we have investigated this issue further by comparing results of geometry optimizations and frequency calculations on the $12\left(d_{\delta}^{A} d_{\delta}^{B}\right)$ states of $\mathrm{Ni}_{2}$ (six singlet, broken symmetry, and six triplet) with B3LYP/AE functional both with and without charge density fitting. The results are summarized in Table V. Although the errors in total energies are rather large (on the order of a little less than $1 \mathrm{mHartree}$, as can be seen in column labeled $E_{e} /$ mHartree in Table V), they all have the same sign, averaging -0.6476 $\pm 0.0018 \mathrm{mHartree}$. Moreover, the errors in the relative energies (with respect to the ground state $\mathrm{Ni}$ atom, labeled $E_{e} / \mathrm{meV}$ in Table $\left.\mathrm{V}\right)$ are much smaller $(\approx 5 \mathrm{meV})$, and again all with the same sign. Finally, the relative ordering of the states is correct, and the root-mean square of the relative energies with respect to the lowest energy state from each calculation, labeled $\Delta E_{e} / \mathrm{meV}$ in Table $\mathrm{V}$, is $0.03 \mathrm{meV}$ with a maximum of $0.12 \mathrm{meV}$. The maximum error due to charge 
TABLE VI. Charge density (CD) fitting errors for the bond lengths $d_{e}$, dissociation energies $D_{e}$, and harmonic vibrational frequencies $\omega_{e}$ of $\mathrm{Ni}_{2}$ and $\mathrm{NiH}$, and dipole moment $\mu$ computed with B3LYP and FSLYP functionals using "Stuttgart RSC ECP" ECP and basis set with "Ahlrichs Coulomb Fitting" basis. The results from the calculations using CD fitting are reported in the "cdfit" columns, the result from the calculations not using CD fitting are reported in "nocdfit" columns, and the differences between the results from the calculations using CD fitting and the results from the ones not using CD fitting are reported under the "cdfit err" columns, with the percent relative errors in parentheses.

\begin{tabular}{|c|c|c|c|c|c|c|c|c|c|c|c|c|c|c|c|c|c|}
\hline \multirow{3}{*}{$\frac{\mathrm{Mol}}{\mathrm{NiH}}$} & \multirow{3}{*}{$\frac{\text { XC }}{\text { B3LYP }}$} & \multicolumn{4}{|c|}{$d_{e}(\AA)$} & \multicolumn{4}{|c|}{$D_{e}(\mathrm{eV})$} & \multicolumn{4}{|c|}{$\omega_{e}\left(\mathrm{~cm}^{-1}\right)$} & \multicolumn{4}{|c|}{$\mu($ Debye $)$} \\
\hline & & \multirow{2}{*}{$\begin{array}{c}\text { nocdfit } \\
1.454\end{array}$} & \multirow{2}{*}{$\begin{array}{l}\text { cdfit } \\
1.456\end{array}$} & \multicolumn{2}{|c|}{ cdfit err } & \multirow{2}{*}{$\begin{array}{c}\text { nocdfit } \\
2.901\end{array}$} & \multirow{2}{*}{$\begin{array}{l}\text { cdfit } \\
2.784\end{array}$} & \multicolumn{2}{|c|}{ cdfit err } & \multirow{2}{*}{$\begin{array}{l}\text { nocdfit } \\
1937.6\end{array}$} & \multirow{2}{*}{$\begin{array}{c}\text { cdfit } \\
1998.8\end{array}$} & \multicolumn{2}{|c|}{ cdfit err } & \multirow{2}{*}{$\begin{array}{c}\text { nocdfit } \\
2.29\end{array}$} & \multirow{2}{*}{$\begin{array}{l}\text { cdfit } \\
2.21\end{array}$} & \multicolumn{2}{|c|}{ cdfit err } \\
\hline & & & & 0.002 & $(0.1)$ & & & -0.117 & $(-4$ & & & 61.2 & $(3$. & & & -0.08 & $(-3$ \\
\hline $\mathrm{NiH}$ & FSLYP & 1.449 & 1.451 & 0.002 & $(0.1)$ & 2.526 & 2.406 & -0.120 & $(-4$ & 1953.4 & 2015.0 & 61.6 & (3.2) & 2.74 & 2.65 & -0.09 & $(-3.3)$ \\
\hline $\mathrm{Ni}_{2}$ & B3LYP & 2.271 & 2.278 & 0.007 & $(0.3)$ & 1.851 & 1.557 & -0.294 & $(-15$ & 269.3 & 255.3 & -14.0 & $(-5.2)$ & & & & \\
\hline $\mathrm{Ni}_{2}$ & FSLYP & 2.236 & 2.241 & 0.005 & $(0.2)$ & 1.325 & 0.999 & -0.326 & $(-24.6)$ & 283.0 & 268.4 & -14.6 & $(-5.2)$ & & & & \\
\hline
\end{tabular}

density fitting to be expected in exploring the PES's of larger clusters is on the order of $2-3 \mathrm{meV}$ per $\mathrm{Ni}$ atom.

As stated in Sec. II, we did not use charge density fitting with ECP because of the large errors that resulted when we tried the use of Ahlrichs Coulomb Fitting basis in combination with Stuttgart RSC ECP. In Table VI we report the errors in the bond lengths $d_{e}$, dissociation energies $D_{e}$, and harmonic vibrational frequencies $\omega_{e}$ of $\mathrm{Ni}_{2}$ and $\mathrm{NiH}$, and dipole moment $\mu$ computed with B3LYP and FSLYP functionals using "Stuttgart RSC ECP" ECP and basis set with "Ahlrichs Coulomb Fitting" basis. The errors in bond lengths are negligible for both $\mathrm{Ni}_{2}$ and $\mathrm{NiH}$, but the errors in the vibrational frequencies of both $\mathrm{Ni}_{2}$ and $\mathrm{NiH}$, diplole moment of $\mathrm{NiH}$ and dissociation energy of $\mathrm{NiH}$, of the order of $5 \%$, are significat. The error in the dissociation energy of $\mathrm{Ni}_{2}$ is large, $-0.3 \mathrm{eV}(-16 \%)$ for B3LYP and $-0.33 \mathrm{eV}(-25 \%)$ for FSLYP.

\section{APPENDIX B: ACCURACY AND CONVERGENCE ISSUES OF DFT COMPUTATIONS}

The numerical integration necessary for the evaluation of the exchange-correlation energy implemented in NWCHEM uses an Euler-MacLaurin scheme for the radial components (with a modified Mura-Knowles transformation) and a Lebedev scheme for the angular components. Table VII lists the grid details for the three levels of accuracy for the numerical integration that are used in our DFT calculations, labeled by the corresponding keywords from NWCHEM (medium, fine, and xfine). In the same table we list convergence criteria used for each level of accuracy of the numerical integration.

In order to assess the errors arising from numerical integration we have performed a series of computations using

TABLE VII. Details of the integration grid for the evaluation of the exchange-correlation energy: the number of atomic radial (rad.) and angular (ang.) shells for $\mathrm{Ni}$ and $\mathrm{H}$, along with the corresponding convergence criteria for the DFT calculations for each level of accuracy of the numerical integration, in atomic units: energy $(E)$, density $(\rho)$ and orbital gradient (orb. grd.).

\begin{tabular}{|c|c|c|c|c|c|c|c|}
\hline \multirow[b]{2}{*}{ Grid } & \multicolumn{2}{|c|}{$\mathrm{Ni}$} & \multicolumn{2}{|c|}{$\mathrm{H}$} & \multicolumn{3}{|c|}{ Accuracy } \\
\hline & rad. & ang. & rad. & ang. & E & $\rho$ & orb. grd. \\
\hline |xfine $\mid$ & 160 & 1454 & 100 & 1202 & $10^{-8}$ & $10^{-7}$ & $10^{-6}$ \\
\hline fine & 130 & 974 & 60 & 590 & $10^{-7}$ & $10^{-6}$ & $10^{-5}$ \\
\hline |medium| & 112 & 590 & 45 & 434 & $10^{-6}$ & $10^{-5}$ & $10^{-4}$ \\
\hline
\end{tabular}

different predefined grid schemes available in NWCHEM. First, we have performed the atomic calculations using both xfine and fine grids. The differences are of the order of total energy target accuracy of the fine grid $(\approx 1.5$ $\times 10^{-7}$ hartree). We have also compared the all-electron DFT computations using B3LYP functional with fine grid against the ones with xfine grid for geometry optimization and frequency calculations for $\mathrm{Ni}_{2},\left(d_{x^{2}-y^{2}}^{A} d_{x y}^{B}\right)$ singlet and triplet states. The differences are on the order of $10^{-4} \AA$ for equilibrium bond length, $2 \times 10^{-6}$ hartree for total equilibrium energy and $0.2 \mathrm{~cm}^{-1}$ for vibrational frequency. We conclude that the fine grid is appropriate for geometry optimization and vibrational frequency calculations, and have used it in the present work. For the PEC scans we use the medium grid, which gives for a 19-point B3LYP/AE PEC scan in the range 2-3.2 $\AA$ of $\mathrm{Ni}_{2}\left(d_{x^{2}-y^{2}}^{A} d_{x y}^{B}\right)$ singlet an error in energy of $16 \mu \mathrm{eV}$ (maximum) and $1.4 \mu \mathrm{eV}$ (root-mean square) with respect to the fine grid computations.

${ }^{1}$ A. W. Castleman, Jr. and R. G. Keese, Annu. Rev. Phys. Chem. 37, 525 (1986).

${ }^{2}$ A. W. Castleman, Jr. and K. H. Bowen, Jr., J. Chem. Phys. 100, 12911 (1996)

${ }^{3}$ M. Moskovits, Annu. Rev. Phys. Chem. 42, 465 (1991).

${ }^{4}$ Clusters Models for Surface and Bulk Phenomena, edited by G. Pacchioni, P. S. Bagus, and F. Parmigiani (Plenum, New York, 1992).

${ }^{5}$ M. B. Knickelbein, Philos. Mag. B 79, 1379 (1999).

${ }^{6}$ D. J. Trevor, R. L. Whetten, D. M. Cox, and A. Kaldor, J. Am. Chem. Soc. 107, 518 (1985).

${ }^{7}$ M. R. Zakin, D. M. Cox, and A. Kaldor, J. Phys. Chem. 91, 5224 (1987).

${ }^{8}$ A. Kaldor, D. M. Cox, and M. R. Zakin, Adv. Chem. Phys. 70, 211 (1988).

${ }^{9}$ M. R. Zakin, D. M. Cox, R. O. Brickman, and A. Kaldor, J. Phys. Chem. 93, 6823 (1989).

${ }^{10}$ D. L. Freeman and J. D. Doll, Annu. Rev. Phys. Chem. 47, 43 (1996).

${ }^{11}$ S. K. Nayak, S. N. Khanna, B. K. Rao, and P. Jena, J. Phys. Chem. A 101, 1072 (1997).

${ }^{12}$ Y. Xiang, D. Y. Sun, and X. G. Gong, J. Phys. Chem. A 104, 2746 (2000).

${ }^{13}$ N. N. Lathiotakis, A. N. Andriotis, M. Menon, and J. Connolly, J. Chem. Phys. 104, 992 (1996)

${ }^{14}$ C. Luo, Modell. Simul. Mater. Sci. Eng. 8, 95 (2000).

${ }^{15}$ E. Curotto, A. Matro, D. L. Freeman, and J. D. Doll, J. Chem. Phys. 108, 729 (1998).

${ }^{16}$ B. V. Reddy, S. K. Nayak, S. N. Khanna, B. K. Rao, and P. Jena, J. Phys. Chem. A 102, 1748 (1998).

${ }^{17}$ C. Bae, Ph.D. thesis, Brown University, 2001.

${ }^{18}$ P. Hohenberg and W. Kohn, Phys. Rev. 136, B864 (1964).

${ }^{19}$ R. G. Parr and W. Yang, Density Functional Theory of Atoms and Molecules (Oxford University Presss, New York, 1989).

${ }^{20}$ D. Cremer, Mol. Phys. 99, 1899 (2001).

${ }^{21}$ H. Basch, M. D. Newton, and J. W. Moskowitz, J. Chem. Phys. 73, 4492 (1980). 
${ }^{22}$ M. Tomonari, H. Tatewaki, and T. Nakamura, J. Chem. Phys. 85, 2875 (1986).

${ }^{23}$ P. Mlynarski and D. R. Salahub, J. Chem. Phys. 95, 6050 (1991).

${ }^{24}$ R. F. Reuse and S. N. Khanna, Chem. Phys. Lett. 234, 77 (1995).

${ }^{25}$ M. C. Michelini, R. P. Diez, and A. H. Jubert, Int. J. Quantum Chem. 70, 693 (1998)

${ }^{26}$ M. C. Michelini, R. P. Diez, and A. H. Jubert, J. Mol. Struct.: THEOCHEM 490, 181 (1999).

${ }^{27}$ M. Castro, C. Jamorski, and D. R. Salahub, Chem. Phys. Lett. 271, 133 (1997).

${ }^{28}$ A. Bérces, Spectrochim. Acta, Part A 53, 1257 (1997).

${ }^{29}$ S. Yanagisawa, T. Tsuneda, and K. Hirao, J. Chem. Phys. 112, 545 (2000).

${ }^{30}$ C. J. Barden, J. C. Rienstra-Kiracofe, and H. F. Schaefer III, J. Chem. Phys. 113, 690 (2000).

${ }^{31}$ A. D. Becke, Phys. Rev. A 38, 3098 (1988).

${ }^{32}$ C. Lee, W. Yang, and R. G. Parr, Phys. Rev. B 37, 785 (1988).

${ }^{33}$ J. P. Perdew, Phys. Rev. B 33, 8822 (1986).

${ }^{34}$ J. P. Perdew, Phys. Rev. B 34, 7046 (1986).

${ }^{35}$ T. Tsuneda, T. Suzumura, and K. Hirao, J. Chem. Phys. 110, 10664 (1999).

${ }^{36}$ J. P. Perdew and Y. Wang, in Electronic Structure of Solids '91, edited by P. Ziesche and H. Eschrig (Akademie, Berlin, 1991).

${ }^{37}$ A. D. Becke, J. Chem. Phys. 98, 5648 (1993).

${ }^{38}$ A. D. Becke, J. Chem. Phys. 98, 1372 (1993).

${ }^{39}$ M. Bernard, J. Chem. Phys. 71, 2546 (1979).

${ }^{40}$ L. Noodleman, J. Chem. Phys. 74, 5737 (1981).

${ }^{41}$ K.-N. Fan, Z. H. Li, W.-N. Wang, H.-H. Huang, and W. Huang, Chem. Phys. Lett. 277, 257 (1997).

${ }^{42}$ R. L. Martin and F. Illas, Phys. Rev. Lett. 79, 1539 (1997).

${ }^{43}$ F. Illas and R. L. Martin, J. Chem. Phys. 108, 2519 (1998).

${ }^{44}$ J. Gräfenstein and D. Cremer, Mol. Phys. 99, 981 (2001).

${ }^{45}$ High Performance Computational Chemistry Group, NwCHEM, A Computational Chemistry Package for Parallel Computers, Versions 4.0.1, 4.1, and 4.5, Pacific Northwest National Laboratory, Richland, Washington 99352, USA, 2001-2003.

${ }^{46}$ W. Kohn and L. J. Sham, Phys. Rev. 140, A1133 (1965).

${ }^{47}$ For the closed shell ${ }^{1} S$ state of Ni, both restricted and unrestricted HF and MP2 calculations are performed and the difference between the restricted and the unrestricted energies is of the same order of magnitude as the convergence criterion $\left(10^{-8}\right.$ Hartree).

${ }^{48}$ H. L. Schmider and A. D. Becke, J. Chem. Phys. 108, 9624 (1998).

${ }^{49} \mathrm{~J}$. Slater, in The Self-Consistent Field for Molecules and Solids, Quantum theory of molecules and solids, Vol. 4 (McGraw-Hill, New York, 1974).

${ }^{50}$ A. J. H. Wachters, Technical Report No. RJ584, IBM, 1969.

${ }^{51}$ A. J. H. Wachters, J. Chem. Phys. 52, 1033 (1970).

${ }^{52}$ P. J. Hay, J. Chem. Phys. 66, 4377 (1977).

${ }^{53}$ C. W. Bauschlicher Jr., S. R. Langhoff, and L. A. Barnes, J. Chem. Phys. 91, 2399 (1989).
${ }^{54}$ M. Dolg, U. Wedig, H. Stoll, and H. Preuss, J. Chem. Phys. 86, 866 (1987).

${ }^{55}$ The basis sets and ECPs in NWCHEM correspond to Revision: Fri Jun 27 1997 of the Stuttgart/Dresden groups.

${ }^{56}$ K. Eichkorn, O. Treutler, M. H. Holger Öhm, and R. Ahlrichs, Chem. Phys. Lett. 240, 283 (1995).

${ }^{57}$ K. Eichkorn, F. Weigend, O. Treutler, and R. Ahlrichs, Theor. Chem. Acc. 97, 119 (1997).

${ }^{58}$ R. W. Warren and B. I. Dunlap, Chem. Phys. Lett. 262, 384 (1996).

${ }^{59}$ A. Szabo and N. S. Ostlund, Modern Quantum Chemistry (Dover, New York, 1996).

${ }^{60}$ R. Paunz, Spin Eigenfunctions (Plenum, New York, 1979).

${ }^{61}$ I. Ciofini and C. A. Daul, Coord. Chem. Rev. 238-239, 187 (2003).

${ }^{62}$ C. J. Cramer, in Essentials of Computational Chemistry. Theories and Models. (Wiley, Chichester, 2002), Chap. 14.4, pp. 456-459.

${ }^{63}$ C. E. Moore, Nat. Bur. Stand. Cir. (U.S.) 467, Vol. II, p. 98 (1952).

${ }^{64}$ J. Sugar and C. Corliss, J. Phys. Chem. Ref. Data 14, Suppl. 2, p. 581 (1985).

${ }^{65}$ R. L. Martin and P. J. Hay, J. Chem. Phys. 75, 4539 (1981).

${ }^{66}$ H.-T. Jeng and C.-S. Hsue, Phys. Rev. B 62, 9876 (2000).

${ }^{67}$ R. Pou-Amérigo, M. Mechán, I. Nebot-Gill, P.-Å. Malmqvist, and B. O. Roos, J. Chem. Phys. 101, 4893 (1994).

${ }^{68}$ J. C. Pinegar, J. D. Langenberg, C. A. Arrington, E. M. Spain, and M. D. Morse, J. Chem. Phys. 102, 666 (1995).

${ }^{69}$ All dissociation energies reported are calculated relative to the energy of the ground state of $\mathrm{Ni}$ atom(s) (without zero-point correction), unless otherwise specified.

${ }^{70}$ H. Wang, H. Haouari, R. Craig, J. R. Lombardi, and D. M. Lindsay, J. Chem. Phys. 104, 3420 (1996).

${ }^{71}$ J. Ho, M. L. Polak, K. M. Ervin, and W. C. Lineberger, J. Chem. Phys. 99, 8542 (1993)

${ }^{72}$ C. W. Bauschlicher, Jr., H. Partridge, and S. R. Langhoff, Chem. Phys. Lett. 195, 360 (1992)

${ }^{73}$ C. W. Bauschlicher, Jr., Chem. Phys. Lett. 246, 40 (1995).

${ }^{74}$ For the exact Hohenberg-Kohn (HK) functional there would be no unrestricted solution, but all of the current approximations of the HK functional suffer from this problem (Ref. 20).

${ }^{75}$ J. A. Gray, M. Li, T. Nelis, and R. W. Field, J. Chem. Phys. 95, 7164 (1991).

${ }^{76}$ P. B. Armentrout and L. S. Sunderlin, in Transition Metal Hydrides, edited by A. Dedieu (VCH, New York, 1992), p. 1.

${ }^{77}$ J. A. Gray, S. F. Rice, and R. W. Field, J. Chem. Phys. 82, 4717 (1985).

${ }^{78}$ C. M. Marian, M. R. A. Blomberg, and P. E. M. Siegbahn, J. Chem. Phys. 91, 3589 (1989). 
The Journal of Chemical Physics is copyrighted by the American Institute of Physics (AIP). Redistribution of journal material is subject to the AIP online journal license and/or AIP copyright. For more information, see http://ojps.aip.org/jcpo/jcpcr/jsp 